\title{
6. Die Ära Berthold Keller (1978-1990)
}

\subsection{Der Kampf um Arbeitsplätze als zweites Aktionsfeld}

Anders als 1963 war 1978 mit dem Wechsel im Vorsitz keine Richtungsänderung in der Programmatik der GTB zu erwarten. Die Personalentscheidung war gut vorbereitet, Buschmann hatte sich in seiner letzten Amtszeit zunehmend auf eine präsidiale Rolle beschränkt und viele Fäden liefen bereits bei Berthold Keller zusammen. Die Mehrheit des sozialpartnerschaftlich orientierten Flügels war gesichert. Die innerorganisatorischen Reformen, die dadurch erreichte finanzielle Stabilität und ein steigender Organisationsgrad waren unbestreitbare Erfolge, doch die Herausforderungen wurden nicht kleiner.

Deutschland erlebte Anfang der 1980er Jahre die nächste Rezession, und zwar mit stark ansteigender Arbeitslosigkeit. Für die GTB kam der verstärkte Druck durch Importe hinzu. Vor diesem Hintergrund stellte der neue Vorsitzende drei Jahre nach seiner Wahl die Frage, ob es durchzuhalten sei, weiter "mit klingendem Spiel ${ }^{1}$ in die Tarifrunden zu ziehen und Vereinbarungen auf dem Niveau des industriellen Durchschnitts zu erzielen. Nicht nur die wirtschaftlichen Rahmenbedingungen waren schlechter, mit dem Ende der sozialliberalen Koalition änderten sich 1982 auch die Vorzeichen in der Politik. Die Arbeitgeber sahen ihr Heil in tarifpolitischer Zurückhaltung, ihr Widerstand gegen gewerkschaftliche Forderungen traf jetzt auf Wohlwollen der Politik. Bundesarbeitsminister Norbert Blüm forderte eine »Lohnpause«. Die Gewerkschaften kämpften um die Sicherung der Reallöhne, also um den Erhalt zumindest der Kaufkraft der abhängig Beschäftigten. Bei der Forderung nach beschäftigungsfördernden Investitionsprogrammen erfolglos geblieben, versuchten die Gewerkschaften, die Arbeitslosigkeit durch Arbeitsumverteilung zu verringern.

1 Keller (1982): Schwierige Zeiten gemeinsam meistern, S. 7. 


\subsubsection{Das GTB-Programm}

Karl Buschmann, der die politische Ausrichtung und das Wirken der GTB entscheidend geprägt hatte, schied 1978 aus Altersgründen als Vorsitzender aus. Bundeskanzler Helmut Schmidt, zu dem Buschmann ein besonderes Vertrauensverhältnis aufgebaut hatte, betonte, wie er dem Gewerkschaftsvorsitzenden gerne und sorgfältig zugehört

»und in mancher Weise seine Ratschläge beherzigt habe. [...] In den fünfzehn Jahren, in denen Karl Buschmann Vorsitzender dieser Gewerkschaft war, ist er zu einem persönlichen Symbol für das geworden, was ich [...] an der deutschen Cewerkschaftsbewegung zu loben versucht habe. ${ }^{2}$

So vom Bundeskanzler gewürdigt ging die Gewerkschaft, die innerhalb des DGB nicht einmal ein Zwanzigstel der Mitglieder einbrachte, selbstbewusst in den Gewerkschaftstag 1978. Die »Wachablösung« beim Vorsitz war gut vorbereitet. Mit Berthold Keller wurde einer der "jungen Wilden«, die den politischen Kurswechsel der GTB in den 1950er Jahren vorangetrieben hatten, Buschmanns Nachfolger. Die Mehrheit der »Godesberger « und Christlich-Sozialen war so stabil, dass sich im Gegensatz zum Wechsel Bock/Buschmann im Jahr 1963 diesmal kein Gegenkandidat auch nur eine Chance ausrechnen konnte, zumal sich Berthold Keller in den Monaten vor dem Gewerkschaftstag profilieren konnte. Die Vorlage dazu lieferte ihm, sicherlich unbeabsichtigt, die Bundesregierung.

Wenige Wochen vor dem Gewerkschaftstag hatte das Bundeswirtschaftsministerium mit einer Anzeige unter dem Titel »Frau Müller und das Hemd aus Ceylon« für die internationale Arbeitsteilung geworben (siehe Kapitel 2.1.5). Sie argumentierte, »an der einfachen Logik, dass andere Länder unsere Maschinen nur bezahlen können, wenn wir ihre Hemden kaufen«, führe kein Weg vorbei. ${ }^{3}$ In der GTB gab es einen Aufschrei, Berthold Keller reagierte in der Mitgliederzeitung empört. Die Beschäftigten der Textil- und der Bekleidungsindustrie hätten den brutalen Preis für diese Art von sogenannter Entwicklungspolitik zu zahlen. Die Arbeiter in Ceylon könnten sich von ihren Löhnen nichts von dem kaufen, was sie produzierten. ${ }^{4}$

Keller organisierte eine Protestkampagne, bei der Betriebsräte Hunderte von Schreiben an die Bundesregierung schickten. Im Vorfeld seiner Wahl hatte er sich damit als unerschrockener Streiter für die Interessen der Beschäftigten

2 GTB: Protokoll des 13. Ordentlichen Gewerkschaftstages, 1.-6. Oktober 1978 in Mannheim, S. 17 und 25 .

3 Zit. nach Keller (1978): Frau Müller und das Hemd aus Ceylon, S. 3.

4 Keller (1978): Frau Müller und das Hemd aus Ceylon, S. 3 f. 
profiliert, der auch vor einer Auseinandersetzung mit der von Helmut Schmidt geführten sozialliberalen Bundesregierung nicht zurückschreckte. Auf dem Gewerkschaftstag 1978 distanzierte sich der Bundeskanzler von der Anzeige und betonte die Notwendigkeit einer eigenständigen und lebensfähigen Textilindustrie. Die Bundesregierung werde dafür sorgen, »daß wir sie behalten $« .^{5}$ Auf demselben Gewerkschaftstag wurde der gelernte Maßschneider Berthold Keller mit einem Ergebnis von 91,3 Prozent (189 von 207 abgegebenen Stimmen) zum Vorsitzenden der GTB gewählt - ein Ergebnis, mit dem er nach eigener Aussage nicht gerechnet hatte und das angesichts der nach wie vor bestehenden politischen Differenzen in der GTB überraschte. ${ }^{6}$

Das Selbstbewusstsein, das sich die Gewerkschaft durch ihr erfolgreiches Krisenmanagement erarbeitet hatte, wollte der neue Vorsitzende auch durch die erstmalige Erarbeitung eines eigenen Programms dokumentieren. Bislang gab es ein umfassendes politisches Programm nur auf DGB-Ebene. Mit dem GTBProgramm versuchte Berthold Keller, der gemeinwohlorientierten Ausrichtung der Textilgewerkschaft ein theoretisches Gerüst zu geben. 1949 hatte die GTB auf dem DGB-Kongress noch eine »echte sozialistische Wirtschaft" gefordert. Dies wird in der Einführung zum neuen "Mannheimer Programm « der verbreiteten "geistigen Position « der damaligen Zeit zugeschrieben. ${ }^{7}$ Seit Anfang der 1960er Jahre hatte die GTB ihre Rolle in der sozialen Marktwirtschaft gesucht und sich unter schwierigsten Rahmenbedingungen organisatorisch und finanziell konsolidiert. Sie war als Sprecherin der Branche anerkannt und legte nun ein »Spiegelbild unseres Selbstverständnisses « vor. ${ }^{8}$

Das Programm mit seinen 149 Punkten in zwölf Kapiteln war eine Mischung aus Zustandsbeschreibung, Grundsätzen und Einzelfragen. Bekräftigt wird der Anspruch, auf der Grundlage von Artikel 9 Absatz 3 Grundgesetz weit mehr tarifvertraglich regeln zu können als die direkten Arbeitsbedingungen. Tarifpolitik müsse umfassender definiert werden:

"Deshalb sollte jede sozial-, wirtschafts- und gesellschaftspolitische Absicht zunächst daraufhin geprüft werden, ob und in welchem Umfang sie durch Tarifvertrag verwirklicht werden kann.«"

Das Prinzip der Einheitsgewerkschaft war ein zentrales Bekenntnis des Programms. Die GTB verpflichtete sich zu den Geboten von Toleranz und gegen-

5 GTB: Protokoll des 13. Ordentlichen Cewerkschaftstages, 1.-6. Oktober 1978 in Mannheim, S. 16.

6 GTB: Protokoll des 13. Ordentlichen Cewerkschaftstages, 1.-6. Oktober 1978 in Mannheim, S. 154.

7 GTB-Programm, in: CTB: informationen für Vertrauensleute 1978, S. 4.

8 CTB: Protokoll des 13. Ordentlichen Cewerkschaftstages, 1.-6. Oktober 1978 in Mannheim, S. 239.

9 CTB: Protokoll des 13. Ordentlichen Gewerkschaftstages, 1.-6. Oktober 1978 in Mannheim, S. 330. 
seitiger Rücksichtnahme bei personellen und sachlichen Entscheidungen und zur Bereitschaft zum Kompromiss. Das Programm kritisierte den durch das Bundesarbeitsgericht geschaffenen hohen Stellenwert der negativen Koalitionsfreiheit und forderte, dass ein verbessertes Tarifvertragsgesetz künftig Differenzierungsklauseln zwischen Mitgliedern und Nichtmitgliedern in Tarifverträgen ermöglichen solle. Einen weiteren Schwerpunkt im Programm bildete das Thema Vermögensbildung, zu dem es hieß:

»[...] eine freiheitlich-demokratische Gesellschaftsordnung ist bei einer derartig ungerechten Verteilung des Produktivvermögens in ihrem Kern gefährdet und bedarf einer umfassenden Beteiligung der Arbeitnehmer an der volkswirtschaftlichen Vermögensbildung. $\ll^{10}$

Die GTB bekannte sich zu einer offenen, pluralistischen Gesellschaft, wie sie der Philosoph Karl R. Popper definiert hatte, und sah sich dem Gemeinwohl verpflichtet. Im Verhältnis zu den Unternehmern betonte sie die Möglichkeit eines »Machtausgleichs « und unterstrich dabei, »die Gewerkschaften erstarrten nie in egoistischer Interessenvertretung ${ }^{11}{ }^{11}$ Somit legte das Programm keinen grundsätzlichen Interessengegensatz zum Kapital zugrunde, sondern ging von der Möglichkeit eines harmonischen Ausgleichs aus. Diese Analyse wurde nicht von allen Gewerkschafter:innen geteilt. Deutliche Kritik wurde insbesondere an der Orientierung am Gemeinwohl laut, da die Unternehmerschaft mit ihrer Klage gegen das Mitbestimmungsgesetz zur gleichen Zeit zum Ausdruck gebracht habe, was sie vom »Machtausgleich « hielt. ${ }^{12}$ Bemerkenswert ist, dass das Programm der Bekämpfung der Arbeitslosigkeit im Allgemeinen und insbesondere des rapiden Arbeitsplatzabbaus in den Bereichen Textil und Bekleidung keinen herausragenden Stellenwert zumisst.

Nach kurzer Diskussion und einigen Gegenstimmen zu den Abschnitten, in denen das Gemeinwohl eine Rolle spielt, wurde das Programm in der folgenden Schlussabstimmung mit nur noch einer Gegenstimme verabschiedet. Bei der Ausarbeitung des Programms waren alle Bezirke intensiv mit eingebunden gewesen, so dass viele verschiedene Anliegen ihren Niederschlag im Programm gefunden hatten. Der politische rote Faden, die Verankerung im gemäßigten Gewerkschaftslager, entsprach den Machtverhältnissen in der Organisation. In dieser Ausrichtung fühlte sich die GTB besonders mit der Gewerkschaft NahrungGenuss-Gaststätten (NGG) verbunden.

10 GTB: Protokoll des 13. Ordentlichen Cewerkschaftstages, 1.-6. Oktober1978 in Mannheim, S. 341.

11 GTB: Protokoll des 13. Ordentlichen Gewerkschaftstages, 1.-6. Oktober 1978 in Mannheim, S. 323.

12 So Bastian (1979): Am Scheideweg, S. 12. 


\subsubsection{Erneutes Ringen um die Welttextilabkommen}

Das erste Welttextilabkommen (WTA) lief Ende 1977 aus (siehe Kapitel 2.1.5). Gegenüber 1973, als der Vertrag verhandelt worden war, hatte sich die wirtschaftliche Lage in Europa stark verändert. Der Nachkriegsboom war zu Ende, nach fast fünfzehn Jahren Vollbeschäftigung waren nun Hunderttausende erwerbslos. Die im WTA festgeschriebenen Wachstumsraten der Textil- und Bekleidungsimporte von jährlich 6 Prozent lagen deutlich über den Steigerungsraten des Textilverbrauchs in Europa, so dass das Abkommen nur eine bescheidene beschäftigungssichernde Wirkung für die deutsche Industrie hatte. Die GTB mobilisierte für eine deutliche Verbesserung des Handelsabkommens und forderte insbesondere eine Sozialklausel, durch die die Lieferländer auf die Einhaltung der Kernarbeitsbedingungen der Internationalen Arbeitsorganisation $\mathrm{ILO}^{13}$ verpflichtet werden sollten. Diese betreffen:

- Vereinigungsfreiheit und Recht auf Kollektivverhandlungen

- Beseitigung der Zwangsarbeit

- Abschaffung der Kinderarbeit

- Verbot der Diskriminierung in Beschäftigung und Beruf

Außerdem sollten für die Hauptlieferländer Hongkong, Südkorea, Taiwan und Macau (von der GTB inoffiziell als »Viererbande« bezeichnet) keine Kontingenterhöhungen erfolgen und die jährlichen Wachstumsraten für die anderen Länder differenziert und grundsätzlich reduziert werden. Eine Rezessionsklausel sollte Änderungen während des laufenden Abkommens ermöglichen und die Verteilung innerhalb der EG stringenter gehandhabt sowie Umgehungseinfuhren begrenzt werden. ${ }^{14}$

Nachdem die GTB ihr Ringen um das erste WTA vor allem auf nationaler Ebene mit der Bundesregierung ausgetragen hatte, stieß sie nun Initiativen auf der europäischen Gewerkschaftsebene an. Nach der Erweiterung der Europäischen Gemeinschaft (EG) im Jahr 1973 hatte der Europäische Gewerkschaftsverband Textil-Bekleidung-Leder (EGV-TBL) 1975 die Gewerkschaften aus Großbritannien, Irland, Norwegen und Dänemark aufgenommen und dadurch ein stärkeres Gewicht. Das Bundeswirtschaftsministerium unter Otto Graf Lambsdorff, grundsätzlich einer liberalen Außenhandelspolitik verschrieben, zeigte sich im Hin-

13 Die ILO ist eine Sonderorganisation der Vereinten Nationen. Die ILO verfügt über eine dreigliedrige Struktur, die im UN-System einzigartig ist: Die 187 Mitgliedsstaaten sind durch Regierungen, Gewerkschaften und Arbeitgeberverbände in den Organen der ILO vertreten; vgl. ILO Vertretung in Deutschland (o.).): Die ILO in Deutschland.

14 textil-bekleidung, Ausgabe 6/1977, S. 3. 
blick auf eine Verbesserung des WTA skeptisch und stand damit auf der Seite der Freihandelsbefürworter. Der stärkste Gegenspieler dieser Position in der EG war Frankreich. Die asiatischen Produktionsländer, an der Spitze Hongkong, drängten auf weitere Erleichterungen. Im Herbst 1977 gerieten die Verhandlungen ins Stocken und im November erklärte Hongkong die Gespräche für gescheitert.

Argumentativ zog die GTB viele Register. Sie versuchte, die Abgeordneten aus textilen Schwerpunktregionen unter Druck zu setzen, und gewann auch die SPD für eine Bundestagsdebatte über ein neues WTA. Dem Wirtschaftsministerium warf sie Frauenfeindlichkeit vor, da die männerdominierte Stahlindustrie gestützt werde, während man die Textil- und die Bekleidungsindustrie im Regen stehenließ.15

Angesichts der Unsicherheit, ob es überhaupt zu einem neuen Abkommen kommen würde, verständigte sich der EGV-TBL darauf, europaweit Druck zu machen. Am 16. November 1977 protestieren Gewerkschafter:innen aus allen EGLändern in Brüssel für ein neues und besseres WTA. Sie forderten die Europäische Kommission auf, bei den Verhandlungen nicht nachzugeben und notfalls ab 1978 einseitige Handelsbeschränkungen einzuführen. Die Gewerkschaften betonten ihre Entschlossenheit, der arbeitsplatzbedrohenden Einfuhrschwemme von Textilien aus Niedrigpreisländern mit aller Kraft entgegenzutreten. ${ }^{16}$ Damit stärkten die europäischen Gewerkschaften die Position von Frankreich, Italien und Großbritannien ${ }^{17}$ und trugen mit ihrem gemeinsamen Auftreten maßgeblich zum Zustandekommen des zweiten WTA bei, das Anfang 1978 mit 31 Lieferländern abgeschlossen wurde. ${ }^{18}$

Die zentrale Forderung nach einer Sozialklausel konnte zwar nicht durchgesetzt werden, dennoch enthielt das neue Abkommen deutliche Verbesserungen. Für sogenannte "hochsensible Erzeugnisse« wie Baumwollgarne und gewebe, Oberhemden und T-Shirts, Pullover, Hosen und Blusen wurden nur geringe Steigerungsraten zugelassen; die Quoten der Hauptlieferländer wurden ganz eingefroren. Die Bundesrepublik sollte nicht mehr als 28,5 Prozent der EG-Importmengen aufnehmen, d.h. die Binnenquoten in Europa wurden verstärkt. Im Laufe des Jahres folgte auch ein Abkommen mit der Volksrepublik China, die bislang kein Vertragspartner des WTA war. Als neuer Player im Welthandel fing China klein an: 1978 kamen von dort nur Importe in Höhe von 131 Millionen DM (67 Millio-

15 Silvia (1990): Jobs, trade and unions, S. 466.

16 textil-bekleidung, Ausgabe 12/1977, S. 3.

17 Frankreich hatte als Reaktion auf die stockenden Verhandlungen schon 1977 einseitige Einfuhrbeschränkungen beschlossen.

18 Silvia (1990): Jobs, trade and unions, S. 469. 
nen Euro ${ }^{19}$, vierzig Jahre später betrug der Wert mit 5,3 Milliarden Euro (2018) ziemlich genau das Vierzigfache. ${ }^{20}$

»Man kann mit Fug und Recht feststellen, daß diese überzeugenden Cewerkschaftsaktionen zum endgültigen Durchbruch und Erfolg führten«,

stellte der GTB-Hauptvorstand zufrieden fest. ${ }^{21}$ Dieser Wertung widersprachen weder die Arbeitgeberverbände noch die Bundesregierung. ${ }^{22}$ Die GTB hatte sich erneut als konsequent im Einsatz für den Schutz der Arbeitsplätze gezeigt. Unter großer Beteiligung ihrer Mitglieder hatte sie mit der Politik auf bundesdeutscher und europäischer Ebene gerungen und dadurch das Thema Beschäftigung auf das politische Feld geschoben.

Damit verschaffte sich die GTB auch in der Tarifpolitik einen größeren Freiraum: Forderten die Arbeitgeber in den Tarifrunden Zugeständnisse angesichts der schwierigen internationalen Wettbewerbssituation, konnte die GTB auf ihren Einsatz für den Schutz der Branche und der hiesigen Arbeitsplätze bei Regierung und EG-Kommission verweisen - und kritisierte zugleich, dass die Arbeitgeber selbst die Produktion in immer stärkerem Maße ins billigere Ausland verlagerten. Der Hauptvorstand forderte die Unternehmer auf, sich verstärkt den Wünschen der Märkte und Verbraucher:innen anzupassen und mit Blick auf Qualität und Mode nachgefragte höherwertige und damit wettbewerbsfähige Erzeugnisse herzustellen. So sei auch ein fester Platz in der heimischen Volkswirtschaft sichergestellt, argumentierte die $\mathrm{GTB},{ }^{23}$ doch dieser Optimismus sollte sich als zu groß erweisen.

\subsubsection{Die gesamtwirtschaftliche Krise verschärft die Branchenkrise}

Der Erste Golfkrieg zwischen dem Irak und Iran führte $1980 \mathrm{zu}$ einem deutlichen Anstieg der Ölpreise, so dass der Ölverbrauch von Konsumgüterartikeln zu Energielieferanten umgelenkt wurde. Die Verbraucher:innen waren verunsichert, der Handlungsspielraum der Bundesregierung durch die Staatverschuldungsdiskussion eingeschränkt. 1981/1982 kam es zur bis dahin schwersten Rezession der Bundesrepublik. Die Arbeitslosenzahl stieg bis an die Zwei-Millionen-Grenze. Die Forderung des DGB nach einem 50-Milliarden-Programm zu Bekämpfung der Arbeitslosigkeit war gegen die FDP nicht durchsetzbar. Von liberaler Seite kamen

19 textil-bekleidung, Ausgabe 6/1978, S. 23.

20 Guinebault (2018): Europas Textilimporte sinken im 1. Halbjahr.

21 GTB: Geschäftsbericht 1974-1977 des Hauptvorstandes, S. 103.

22 Silvia (1990): Jobs, trade and unions, S. 470.

23 GTB: Geschäftsbericht 1974-1977 des Hauptvorstandes, S. 106. 
stattdessen Forderungen nach Lohnverzicht, Kürzung des Arbeitslosengeldes und Wiedereinführung von Karenztagen im Krankheitsfall.

Die GTB beklagte, dass Regierung und Sachverständigenrat auf Investitionen setzten, um damit die Volkswirtschaft zu modernisieren und die internationale Wettbewerbsfähigkeit zu steigern. Ihre Befürchtung aus der Erfahrung der vergangenen Jahre war, dass Maschinenexporte als »Gegenleistung « weitere Textilimporte nach sich ziehen würden. Die inländischen Frauenarbeitsplätze würden gefährdet, Ersatz gebe es nicht. ${ }^{24}$ In den Jahren 1977 bis 1980 hatte sich der Arbeitsplatzabbau bei Textil und Bekleidung verlangsamt, im Zuge der Wirtschaftskrise zog er wieder an. 1980 bis 1983 gingen erneut über 100.000 Arbeitsplätze in diesen Sektoren verloren. Von 1970 bis 1984 waren die Beschäftigtenzahlen auf 430.000 gesunken und hatten sich damit mehr als halbiert. Das Statistische Bundesamt meldete für denselben Zeitraum den Rückgang um 4.100 Betriebe auf nur noch 4.700 Produktionsstätten. Auch diese Wirtschaftskrise bestätigte, dass gesamtwirtschaftliche Verwerfungen die Branchen Textil und Bekleidung überproportional treffen, weil der Konsum textiler Produkte deutlich zurückgefahren oder auf billigere Importware zurückgegriffen wird.

Neben der DGB-Forderung nach einem mittelfristigen Beschäftigungsprogramm setzte die GTB einen weiteren Schwerpunkt ihrer Arbeit im Ringen um die Verbesserung des 1981 auslaufenden zweiten Welttextilabkommens (WTA; siehe Kapitel 6.1.2). Erstmals betonte sie auch die unterschiedlichen Wettbewerbsbedingungen der Textil- und der Bekleidungsbranche innerhalb der EG. Während Italien und Frankreich diese Branchen direkt und indirekt subventionierten und damit förderten, überlasse die Bundesregierung die Industrien allein dem Markt. Im Vordergrund stand weiterhin die Durchsetzung eines besseren WTA. Die negative Beschäftigungsentwicklung sei kein unabwendbares Schicksal, das man einfach hinnehmen werde, hieß es selbstbewusst. ${ }^{25}$ Seit 1980 warb die GTB im Verbund mit ihren europäischen Schwesterorganisationen

- für ein Abkommen mit Import-Steigerungsraten unter 1,5 Prozent jährlich,

- für eine mindestens zehnjährige Laufzeit, um Sicherheit für Investitionen zu gewährleisten,

- für die Kürzung der Quoten für bereits industrialisierte Entwicklungsländer zugunsten ärmerer Staaten,

- für bessere Kontrolle der Binnenverteilung innerhalb der EG und

24 GTB: Geschäftsbericht 1978-1981 des Hauptvorstandes, S. 60.

25 GTB (1991): textil-bekleidung. 100 Jahre GTB. Sonderausgabe zum Jubiläum, S. 158. 
- für die Einhaltung der ILO-Kernarbeitsnormen als Voraussetzung für einen fairen Handel. ${ }^{26}$

Die europäische Kommission war zunächst nicht für eine Verlängerung des WTA zu gewinnen. Insbesondere wiesen die EG-Verantwortlichen die Gewerkschaftsvertreter:innen auf die Haltung des deutschen Wirtschaftsministers Graf Lambsdorff hin, der das Auslaufen des Schutzabkommens vertrete. ${ }^{27}$ Die GTB reagierte zunächst mit Unterschriftenaktionen und Briefen an die Mitglieder des Bundestages; schließlich beschlossen die europäischen Textil-BekleidungsGewerkschaften eine gemeinsame europaweite Arbeitsniederlegung. Erstmals in der Geschichte der europäischen Gewerkschaften wurden die Beschäftigten länderübergreifend dazu aufgerufen, am 2. Dezember 1980 die Arbeit von 11 bis 12 Uhr ruhen zu lassen, um für ein besseres Schutzabkommen für die Branchen zu demonstrieren. Die Aktion war erfolgreich, 2,5 Millionen Arbeitnehmer:innen beteiligten sich an dem Streik. Diese hohe Mobilisierung wurde dadurch erleichtert, dass die Mehrheit der Arbeitgeber die Aktion unterstützte oder zumindest tolerierte. In Deutschland legten am Protesttag 275.000 Beschäftigte die Arbeit nieder. ${ }^{28}$

Der millionenfache Protest löste ein Medienecho aus und verfehlte seine Wirkung nicht. Die EG-Kommission schwenkte um und auch der deutsche Bundeskanzler Helmut Schmidt setzte sich nun für ein besseres WTA ein. ${ }^{29}$ Dennoch blieb der deutsche Wirtschaftsminister bei der Erarbeitung einer gemeinsamen Linie der europäischen Länder der Störenfried. Aus diesem Grund organisierte die GTB 1981 eine Kampagne unter dem Titel »Mein Arbeitsplatz muss bleiben«. In einer ganzen Reihe von regionalen Kundgebungen wurde Druck auf Lambsdorff aufgebaut: Es gebe staatliche Hilfen für Kohle, Stahl und den Schiff- und Flugzeugbau, aber die Frauenbranchen Textil und Bekleidung würden geopfert. ${ }^{30}$

Schließlich entschloss sich der Hauptvorstand, zum zweiten Mal zu einer Massenkundgebung gegen die Textil-Handelspolitik der sozialliberalen Regierung aufzurufen. 20.000 Beschäftigte folgten dem Aufruf und demonstrieren am 21. November 1981 auf dem Bonner Münsterplatz für den Erhalt ihrer Arbeitsplätze. Wie schon 1973 bei der ersten bundesweiten Protestkundgebung war die Politik alarmiert. Der Bundeskanzler empfing Berthold Keller und sicherte seine Unterstützung bei den Verhandlungen zu; der Bundestag forderte in einer Reso-

26 textil-bekleidung, Ausgabe 12/1980, S. 7 (Cewerkschaftsforderungen zum Welttextilabkommen).

27 CTB: Ceschäftsbericht 1978-1981 des Hauptvorstandes, S. $115 \mathrm{ff}$.

28 GTB: Geschäftsbericht 1978-1981 des Hauptvorstandes, S. 86.

29 Silvia (1990): Jobs, trade and unions, S. 503.

30 GTB: Ceschäftsbericht 1978-1981 des Hauptvorstandes, S. $69 \mathrm{ff}$. 
lution die Verlängerung des WTA und übernahm dabei im Wesentlichen die GTBForderungen. ${ }^{31}$

Die veränderte Haltung der Bundesrepublik führte dazu, dass die EG buchstäblich in letzter Minute eine Übergangsverlängerung vereinbarte. Im Februar 1982 stand dann das neue Abkommen: Hongkong und Taiwan mussten ihre Exporte in die EG reduzieren, bei hochsensiblen Produkten wurde die Importsteigerung auf 1 bis 2 Prozent im Jahr begrenzt, bei sensiblen Produkten auf 4 Prozent. Bei anderen Textil- und Bekleidungswaren wurden Steigerungsraten bis zu 6 Prozent jährlich ermöglicht. Erstmals wurde im dritten WTA neben der wirtschaftlichen auch die soziale Entwicklung der Lieferländer als Hauptziel genannt.

Nach den ersten beiden Abkommen feierte die GTB auch das dritte WTA als einen Erfolg, den sie sich zu großen Teilen auf ihre Fahnen schrieb. Im Ringen um das Abkommen hatte sie ihre Mobilisierungsfähigkeit auch auf dem Feld wirtschaftspolitischer Fragen deutlich gemacht. Der Hauptvorstand hatte sich als Kämpfer für die Arbeitsplätze seiner Mitglieder erwiesen, was die Gewerkschaft spürbar festigte. Auch wenn die GTB in den Jahren 1980 bis 1983 ihren Mitgliederstand nicht halten konnte, gelang es ihr durch die hohe Mobilisierung immerhin, in diesen vier Jahren 125.882 Mitglieder neu zu gewinnen. 1983 hatte sie mit 50,8 Prozent aller Beschäftigten in ihren Branchen den historisch höchsten Organisationsgrad erreicht. ${ }^{32}$ Im DGB-Durchschnitt betrug der Höchststand beim Verhältnis der Gewerkschaftsmitglieder zu den abhängig Beschäftigten insgesamt 39 Prozent im Jahr 1980. ${ }^{33}$

\subsubsection{Neue Wege in der Tarifpolitik: Sparlohn statt Barlohn?}

Zu Jahresbeginn 1982 betonte Berthold Keller, dass die steigende Arbeitslosigkeit und der überproportionale Beschäftigungsrückgang in den Branchen neue, machbare Antworten erforderten. Damit stellte die GTB nun einen Zusammenhang zwischen Arbeitsmarkt- und Tarifpolitik her. In der Januarausgabe der Mitgliederzeitschrift schrieb Keller:

»Die Zeit ist offensichtlich um, in der wie bisher gewohnt mit klingendem Spiel der jahrzehntelang gewohnte Tarifmarsch angetreten werden kann. ${ }^{34}$

Unter dem Motto »Tarifliche Zukunftsvorsorge« warb die GTB dafür, das Thema Vermögensbildung mit Arbeitsplatzsicherheit und Lohnpolitik zu verbinden. Die

31 GTB: Geschäftsbericht 1978-1981 des Hauptvorstandes, S. 118f.

32 Angaben laut Geschäftsberichten des GTB-Hauptvorstandes.

33 Greef (2014): Gewerkschaften im Spiegel von Zahlen, Daten und Fakten, S. 699.

34 Keller (1982): Schwierige Zeiten gemeinsam meistern, S. 7. 
Lohnerhöhungen könnten zukünftig zweigeteilt sein: Der eine Teil werde wie bisher als Barlohn ausbezahlt, ein anderer Teil verbleibe als Investivlohn im Unternehmen oder einem Branchenfonds. Die Industrie müsse eine ausreichende Kapitalausstattung haben, wenn sie im immer härter werdenden internationalen Konkurrenzkampf überleben soll. Mit dieser »tarifpolitischen Vorwärtsstrategie«, wie Keller die Idee bezeichnete, sollte der schwierigen Lage entsprochen werden, ohne eine Abkopplung von der allgemeinen Lohnentwicklung hinnehmen zu müssen. ${ }^{35}$

Angesichts der schwierigen wirtschaftlichen Lage bei Textil und Bekleidung sah es Keller als zunehmend unmöglich an, in der Tarifentwicklung mit anderen Branchen Schritt zu halten. Mit Hilfe eines Investivlohnmodells hielt er die Durchsetzung des gleichen Volumens jedoch für möglich, wenn ein Teil der Lohnerhöhung nicht zur Auszahlung kommt, sondern als Kredit im Unternehmen verbleibt. So könne man einer dauerhaften Abkopplung der textilen Tariferhöhungen von der allgemeinen Einkommensentwicklung entgegenwirken und zugleich Arbeitsplätze durch die verbesserte Liquidität der Unternehmen sichern. ${ }^{36}$ Für Keller stand angesichts der steigenden Arbeitslosigkeit Anfang 1982 fest:

»Alle Cruppen in unserer Cesellschaft werden bereit sein müssen, ihren angemessenen Beitrag zur umfassenden Sicherung unserer demokratischen Gesellschaft zu leisten. Diese Forderung gilt auch für die Gewerkschaften. Auch wir werden umdenken müssen. « $^{37}$

Damit setzte der GTB-Vorsitzende andere Akzente als Eugen Loderer, der Vorsitzende der IG Metall, der die »kapitalistische Wirtschaftsordnung « anders bewertete und einen grundsätzlichen Politikwechsel, nämlich eine demokratischere Kontrolle der Wirtschaft forderte:

»Praktische Erfolge im Kampf gegen die Arbeitslosigkeit und für Vollbeschäftigung erfordern die schrittweise Einschränkung der alleinigen unternehmerischen Verfügungsgewalt über Produktion und Preise, über Investitionen und Arbeitsplätze. ${ }^{38}$

Wie das unter den gegebenen politischen Mehrheitsverhältnissen auch nur mittelfristig erreicht werden könne, ließ er allerdings offen.

35 GTB: Geschäftsbericht 1978-1981 des Hauptvorstandes, S. 61.

36 GTB: Geschäftsbericht 1978-1981 des Hauptvorstandes, S. 62.

37 Keller (1982): Herausforderungen der 8oer Jahre für die Gewerkschaft Textil-Bekleidung, S. 40.

38 Loderer (1982): Für eine arbeitsorientierte Wirtschaftspolitik, S. 18. 
Der Gewerkschaftstag der GTB im Oktober 1982 sollte den Weg für das Konzept des Investivlohns freimachen. Die Idee des Vorsitzenden wurde von der Mehrheit im Hauptvorstand getragen, aber im Vorfeld des Gewerkschaftstages wurde deutlich, dass das Konzept ohne Einbezug der Bezirke und Bezirkstarifkommissionen entwickelt worden war: Es kollidierte schlichtweg mit der Lebenswirklichkeit vieler betrieblicher Funktionär:innen, die gegen die von »ihren « Unternehmen betriebene Produktionsverlagerung und Personalreduzierung kämpften. Auch die Erfahrung mit Firmenpleiten, bei denen die Werte wie Grundstücke, Gebäude und Produktionsanlagen »rechtzeitig« ausgegliedert worden waren, so dass die Beschäftigten leer ausgingen, spielten eine entscheidende Rolle. Außerdem wandten die Kritiker:innen des Modells ein, dass die Unternehmer eigenmächtig über die Verwendung des Kapitals entschieden und die Mittel zum Ausbau von Auslandsproduktionen verwendet würden.

Bei der Vorstellung seines Investivlohnkonzeptes stieß der Hauptvorstand auf so starke Skepsis, dass er den Kritiker:innen schrittweise entgegenkam, bis der Antrag auf dem Gewerkschaftstag schließlich »weichgespült« war. ${ }^{39}$ Die Abstimmungsvorlage sah vor, dass ein entsprechender Tarifvertrag »zur Zukunftsvorsorge und Arbeitsplatzerhaltung « nicht vom Hauptvorstand, sondern nur von den bezirklichen Tarifkommissionen abgeschlossen werden kann. Teile der Lohnund Gehaltserhöhung würden demnach als Darlehen im Unternehmen verbleiben, wobei diese Anteile auf maximal zehn Jahre festliegen und verzinst sowie gegen Verlust abgesichert sein sollten. ${ }^{40}$

Berthold Keller betonte während der Antragsbegründung auf dem Gewerkschaftstag, dass rechtlich noch viele Fragen zu klären seien, so dass die Annahme des Antrages noch keine Entscheidung darüber sei, ob der Investivlohn tarifpolitisch angegangen werden solle. Den Skeptiker:innen kam er durch eine weitere Änderung entgegen, wonach eine Tarifkonzeption, die einen solchen Tarifvertrag umfasse, einer Zweidrittelmehrheit im Hauptvorstand und im Beirat bedürfe. Trotz der zahlreichen Zugeständnisse kam es zu einer grundsätzlichen Debatte. Die Gegner:innen bestritten nicht nur die Wirkung, sie befürchteten sogar negative Auswirkungen für die Beschäftigten:

»Nachgeworfene Grundstücke, Investitionszulagen, Steuergeschenke, billige, brave, fleißige Mitarbeiter - all diese Dinge konnten die Unternehmer nicht daran hindern [, die Produktion ins Ausland] zu verlagern. Die Produktivität wird ständig

39 Vgl. auch die Mitgliederzeitschrift »textil-bekleidung«, in der die Redaktion 1982 in mehreren Ausgaben Leserbriefe pro und kontra Investivlohn einander gegenüberstellte.

40 GTB: Protokoll des 14. Ordentlichen Gewerkschaftstages, 17.-22. Oktober 1982 in Mainz, S. $389 f$. 
gesteigert, die Produkte sind aber nicht absetzbar, weil nicht genügend Kaufkraft vorhanden ist. « $^{41}$

Die Befürworter:innen warnten davor, die Tür für dieses Konzept endgültig zuzuschlagen. Angesichts der gravierenden Arbeitsplatzverluste dürfe man neue Wege nicht von vornherein ausschließen und sich selbst ein mögliches Werkzeug verbieten. ${ }^{42}$ Nachdem das Thema schon in der Debatte zu den Geschäftsberichten eine herausragende Rolle gespielt hatte, wurde die Debatte bei der Antragsberatung nach nur zwölf Redebeiträgen geschlossen, obwohl noch 26 weitere Wortmeldungen vorlagen. Die geheime Abstimmung ergab 121 Ja-Stimmen, 78 Delegierte stimmten mit Nein, 2 enthielten sich. ${ }^{43}$ Mit dieser Dreifünftelmehrheit war die Debatte beendet, zugleich aber das Konzept gestorben. Angesichts der starken Kritik und des nicht wirklich überzeugenden Abstimmungsergebnisses stellte keine Tarifkommission jemals einen Antrag auf eine entsprechende Tarifforderung.

Unter Verantwortung von Berthold Keller wurden in den Tarifrunden 1979 und 1980 unabhängig von der schwierigen Beschäftigungssituation Tariferhöhungen von rund 4,5 Prozent und knapp 7 Prozent erzielt und damit Reallohnzuwächse erreicht, die mehr als ein halbes Prozent über dem Durchschnitt aller Tarifabschlüsse in der Bundesrepublik lagen. ${ }^{44} 1981$ hielten die tariflichen Lohnund Gehaltserhöhungen erstmals nicht mit der Inflationsentwicklung Schritt, in der schweren Rezession sanken die Realeinkommen.

Im Herbst 1982 wurde Bundeskanzler Helmut Schmidt durch ein konstruktives Misstrauensvotum von Helmut Kohl gestürzt. Die FDP hatte sich umorientiert, der Wirtschaftsminister 1981 mit seinem »Lambsdorff-Papier« quasi den Scheidungsantrag vorgelegt. Die Forderungen des Wirtschaftsministers orientierten sich an der Politik der USA unter Ronald Reagan und Großbritanniens unter Margaret Thatcher und sind als erster Vorstoß zu werten, eine neoliberale Wende in der Wirtschafts- und Sozialpolitik einzuleiten. Die Regierung Kohl kündigte Einschnitte ins soziale Netz an; der neue Arbeitsminister Norbert Blüm forderte zu einer »Lohnpause « auf, um die Wirtschaft wieder zu beleben, und manifestierte damit die Rahmenbedingungen für die Tarifrunde 1983.

Die Arbeitgeberverbände versuchten diese regierungsseitige Unterstützung zu nutzen. Der Geschäftsbericht der GTB spricht von »einem konzentrierten An-

41 So die Delegierte Doris Trühe, zit. nach: CTB: Protokoll des 14. Ordentlichen Gewerkschaftstages, 17.-22. Oktober 1982 in Mainz, S. 301.

42 So Wilhelm Werner, Bezirksleiter Baden-Württemberg, zit. nach: GTB: Protokoll des 14. Ordentlichen Cewerkschaftstages, 17.-22. Oktober 1982 in Mainz, S. 303.

43 CTB: Protokoll des 14. Ordentlichen Gewerkschaftstages, 17.-22. Oktober 1982 in Mainz, S. 313.

44 Beyer (1991): Tarifpolitik in strukturschwachen Branchen, S. 171. 
griff der Arbeitgeber-Phalanx auf vielfältige, tariflich geregelte Arbeitsbedingungen«. Hermann Schumacher, seit zwanzig Jahren im GHV für die Tarifpolitik zuständig, kritisierte die Arbeitgeber, die neben Verschlechterungen von Urlaubsund Urlaubsgeldabkommen auch die "nahtlose Anpassung der Arbeitnehmer an die betrieblichen Erfordernisse durch eine flexible Arbeitszeitgestaltung und die tarifliche Absegnung von Samstagsarbeit zu unannehmbaren Bedingungen« forderten. So könnten auch die "gegen den härtesten Widerstand der Arbeitgeber durchgesetzten Verdienststeigerungen" nicht ausreichen, um den Reallohn zu sichern. ${ }^{45}$

Trotz Warnstreiks konnten die Preissteigerungen mit dem 1983 erzielten Tarifergebnis nicht ausgeglichen werden. Dies war jedoch kein GTB-Phänomen, auch den anderen Gewerkschaften gelang es nicht, in ihren Tarifrunden Reallohnsteigerungen durchzusetzen. Dennoch hielt die GTB trotz wesentlich schlechterer wirtschaftlicher Branchenbedingungen mit dem Durchschnitt aller Tariferhöhungen mit. ${ }^{46}$

Der Gedanke, unter schwierigen wirtschaftlichen Bedingungen neue Wege der Tarifpolitik zu gehen, hätte eine breitere Diskussion verdient gehabt. Jahrzehnte später kam er im Zusammenhang mit Verzichtsforderungen an Arbeitnehmer:innen mehrfach wieder zum Vorschein. Ob in der Flächentarifpolitik oder in Tarifverträgen mit Abweichungen für einzelne Firmen, in der Regel waren die von den Gewerkschaften akzeptierten Verluste endgültig. Die IG Metall verzeichnete im Jahr 2007 bei 6.000 Betrieben im Flächentarifvertrag über 1.000 ergänzende, meist die Tarifleistungen mindernde Vereinbarungen. Den Weg dazu hatte sie 2004 mit dem Tarifabkommen von Pforzheim offiziell anerkannt und damit geöffnet.

Besonders in der Weltwirtschaftskrise 2008/2009 wurden Modelle diskutiert, Zugeständnisse bei den Entgelten in Beteiligungen oder Darlehen umzuwandeln, ohne dass dabei tragfähige Lösungsmodelle entwickelt werden konnten oder gar Beispiele geschaffen wurden. Kellers entsprechender Vorstoß von 1982 scheiterte früh an der mangelnden Beteiligung der wichtigen Bezirkstarifkommissionen und der fehlenden Offenheit bei der Entscheidungsfindung.

\subsubsection{Mitgliederinteresse statt Vertragstreue?}

Nach der Rezession im Jahr 1982 kam die deutsche Wirtschaft wieder auf Wachstumskurs, die Zuwachsraten blieben aber zwischen 1,6 und 2,4 Prozent und damit bescheiden. Die Umsätze der Textil- und der Bekleidungsindustrie entwickelten sich positiver als die Gesamtwirtschaftsleistung; der Beschäftigungsabbau in

45 CTB: Geschäftsbericht 1982-1985 des Hauptvorstandes, S. 443 (alle Zitate).

46 Beyer (1991): Tarifpolitik in strukturschwachen Branchen, S. 171. 
diesen Branchen ging auf unter 2 Prozent jährlich zurück. Im Jahr 1988 stieg das Wachstum des Bruttoinlandsprodukts in der Bundesrepublik wieder über die 3-Prozent-Marke. Diese Entwicklung setzte sich 1989 mit 3,9 Prozent fort.

Im Frühjahr 1988, als der Wirtschaftsboom der Folgejahre noch nicht vorauszusehen war, wurde im Zusammenhang mit einer Wochenarbeitszeitverkürzung ein Dreijahres-Tarifvertrag abgeschlossen, der neben dem Lohnausgleich Lohnund Gehaltserhöhungen in Höhe von 3,6 Prozent für 1988 und 1,2 bzw. 2,o Prozent für die beiden Folgejahre vorsah. Unter Berücksichtigung der Preissteigerungsrate von 2,8 Prozent für 1989 und der erwarteten weiter guten Wirtschaftsentwicklung für 1990 würde folglich die reale Kaufkraft wieder sinken. Angesichts der ausgezeichneten Auftragslage bei Textil und bei Bekleidung wuchs bereits 1989 die Unzufriedenheit der Beschäftigten.

Geprägt von den Erinnerungen an die spontanen Streiks des Jahres 1969 (siehe Kapitel 4.1.7) begann die GTB-Spitze Ende 1989 Gespräche mit den Arbeitgeberverbänden, um eine Verbesserung des Tarifergebnisses von 1988 zu erreichen. Die Arbeitgeber, selbst von den starken Auftragseingängen überrascht, wollten die Gelegenheit nutzen, die vereinbarten Arbeitszeitverkürzungen aufzuheben oder zumindest aufzuschieben. Dabei unterschätzten sie, dass die GTB den hart erkämpften Einstieg in die 35-Stunden-Woche nicht anrühren konnte, ohne ihre Glaubwürdigkeit zu verlieren. Die Arbeitgeber waren die Gewinner der 1988 abgeschlossenen Tarifwette auf die zukünftige wirtschaftliche Entwicklung.

Diesen Gewinn wollten die Arbeitgeberverbände der Textil- und der Bekleidungsindustrie behalten. Während beispielsweise die Beschäftigten im Öffentlichen Dienst noch im laufenden Tarifvertrag höhere Zuschläge und damit eine deutliche Einkommensverbesserung erhielten, waren die Arbeitgeber der Textilund der Bekleidungsindustrie zu solchen Zugeständnissen nicht bereit. Verträge seien einzuhalten und vereinbarte lange Laufzeiten nun mal mit Risiken für beide Seiten verbunden.

Angesichts der wachsenden Unruhe insbesondere unter den Funktionär:innen in den größeren Betrieben beschloss die GTB im März 1990 einen anderen Weg, um die Einkommen zu verbessern. Die Tarifverträge über die Jahressonderzahlungen (Weihnachtsgeld) waren kündbar. Je nach Tarifgebiet garantierten sie bislang 40 bis 65 Prozent des dreizehnten Monatseinkommens. Auf Vorschlag des Hauptvorstandes beschloss der Beirat im März 1990 die Kündigung dieser Verträge mit dem Ziel, ein volles dreizehntes Monatsentgelt zu vereinbaren.

Die Arbeitgeberseite wertete das als Vertragsbruch, hatte man sich doch 1988 auf ein Ergebnis für drei Jahre verständigt. Ihre Empörung über das Vorgehen der Gewerkschaft drückten die Arbeitgeberverbände dadurch aus, dass sie die üblichen Bestätigungen über den Eingang der Kündigungsschreiben demonstrativ verweigerten. Formal war der Vorwurf des Vertragsbruchs falsch, da die betreffenden Tarifverträge kündbar waren. Tarifpolitisch und auch mit Blick auf 
ihr sozialpartnerschaftliches Grundverständnis wurde diese eingeschobene Tarifrunde für die GTB zum Balanceakt. Dies macht die »Rechtfertigung« von Wolfgang Stender deutlich, der im Hauptvorstand für Tarifpolitik zuständig war und in der Mitgliederzeitung schrieb:

»Die Tarifforderung der Cewerkschaft Textil-Bekleidung für 1990 ist kein Nachschlag! [...] Wir halten uns an die dreijährige Laufzeit [...]. Die Erhöhung der Jahressonderzahlung auf 100 Prozent ist eine eigenständige tarifliche Forderung, deren Regelung längst überfällig geworden ist. [...] Es geht darum, noch in diesem Jahr etwas zur Verbesserung der Einkommenssituation [...] zu tun. [...] Die Arbeitgeberseite will es offensichtlich nur zur Kenntnis nehmen, daß wir von gesamtwirtschaftlichen und tarifpolitischen Entwicklungen überrollt werden, auf die reagiert werden muss. ${ }^{47}$

Der Druck auf die GTB aus dem Umfeld war immens. Während aus dem 1988er Tarifabschluss für Textil und Bekleidung eine Verbesserung von 2 Prozent anstand, wurden in regulären Tarifrunden bis Mitte Mai 1990 Einkommensverbesserungen von 6,0 Prozent für die Metallindustrie, 6,3 Prozent für die Bauwirtschaft und 6,8 Prozent für die Druckindustrie erreicht. Nachdem die GTB auch für die Großwäschereien einen Tarifabschluss mit einer 5,5-prozentigen Lohnerhöhung erzielt hatte, wurden für Ende Mai erste Verhandlungstermine für die Textilindustrie vereinbart, ${ }^{48}$ die jedoch erfolglos blieben. Auch eine Warnstreikwelle brachte keine Bewegung in die Verhandlungen.

Deshalb legten 15.000 Beschäftigte in Baden-Württemberg für vier Stunden die Arbeit nieder. In den nächsten Tagen sollte über einen unbefristeten Streik abgestimmt werden, woraufhin die Arbeitgeber in diesem Tarifbezirk ein Angebot vorlegten. Am 4. Juli wurde vereinbart, dass die Löhne und Gehälter ab November 1990 um 2,7 Prozent angehoben werden, außerdem erhielten alle Arbeiter:innen und Angestellten bereits mit der Juli-Abrechnung eine Einmalzahlung von 440 DM. Im Gegenzug akzeptierte die GTB die Möglichkeit einer flexibleren Verteilung der Wochenarbeitszeit: Anstatt jede Woche 38,5 Stunden zu arbeiten, musste diese durchschnittliche wöchentliche Arbeitszeit künftig innerhalb von dreizehn Wochen erreicht werden. ${ }^{49}$

Diese »Nachbesserung« des gültigen Tarifvertrages war ein beachtlicher Erfolg, auch wenn sich die geforderte Anhebung der Jahressonderzahlung nicht im Tarifabschluss wiederfand. Der baden-württembergische Verband Südwesttextil hatte unter der Streikdrohung angeboten, was die Arbeitgeber zunächst vehe-

47 Stender (1990): Konflikt um jeden Preis?, S. 3.

48 textil-bekleidung, Ausgabe 6/1990, S. 6 f.

49 GTB: Geschäftsbericht 1990-1993 des Hauptvorstandes, S. 286. 
ment abgelehnt hatten, nämlich eine Tariferhöhung während der eigentlich noch sechs Monate geltenden Laufzeit. Doch die übliche Übertragung des Ergebnisses auf die anderen Bezirke erfolgte nicht.

Deren Tarifkommissionen bestanden auf der Erhöhung der Jahressonderzahlung, um ein volles dreizehntes Monatseinkommen zu sichern, allen voran der streikerfahrene Bezirk Nordrhein. Hier folgte schon zwei Wochen später der nächste Tarifabschluss, der sich an der Forderung orientierte und sie in einem Stufenplan umsetzte: Im Jahr 1990 wurde die Jahressonderzahlung um 25 Prozentpunkte auf 90 Prozent eines Monatseinkommens, in den beiden Folgejahren jeweils um weitere fünf Prozentpunkte erhöht, so dass das volle dreizehnte Monatseinkommen 1992 erreicht wurde. ${ }^{50}$ Ende August wurden diese Erhöhungsstufen schließlich auch für die Bekleidungsindustrie durchgesetzt. ${ }^{51}$

Nach dieser für die Arbeitgeber leidvollen Erfahrung bestanden sie bei allen folgenden Tarifabschlüssen auf dem Zusatz, dass die materiellen Belastungen der Unternehmen für die Laufzeit der Lohn- und Gehaltstarifverträge abschließend geregelt sind. Befördert wurde die Tarifeinigung durch die noch ein Jahr zuvor unabsehbaren Entwicklungen in Deutschland: Die DDR befand sich in Auflösung, wovon die westdeutsche Wirtschaft in hohem Maße profitierte.

\subsection{Arbeitszeitverkürzung und ein besseres Welttextilabkommen sollen die Beschäftigung sichern}

Nach der Ablösung der sozialliberalen Regierung durch die Regierung Kohl/Genscher im Jahr 1982 stieg die Arbeitslosigkeit weiter von 7,5 Prozent auf 9,1 Prozent im Jahr 1983. Die neue von CDU/CSU und FDP geführte Bundesregierung ging auf gewerkschaftliche Forderungen nach einer umfangreichen Intervention zur Belebung der Wirtschaft nicht ein. Die Gewerkschaften setzten auf das Instrument der Arbeitszeitverkürzung, das sie autonom über Tarifverträge erreichen konnten, um Beschäftigung zu sichern und auf mehr Köpfe zu verteilen. Mit diesem Ansatz ernteten sie heftigen Widerspruch von Arbeitgebern und Regierung. Beide sahen die Wettbewerbsbedingungen der bundesdeutschen Wirtschaft durch die steigende Kostenbelastung aufgrund verkürzter Arbeitszeiten in Gefahr.

50 GTB: Geschäftsbericht 1990-1993 des Hauptvorstandes, S. 287.

51 GTB: Geschäftsbericht 1990-1993 des Hauptvorstandes, S. 302. 


\subsubsection{5-Stunden-Woche oder Vorruhestand?}

Der neuen Bundesregierung gelang es nicht, die Arbeitslosigkeit durch Änderungen bei den angeblich die Beschäftigung hemmenden Arbeitnehmerschutzrechten wirksam zu bekämpfen. 1983 stieg die Zahl der Arbeitslosen nochmals um eine halbe Million auf insgesamt 2,3 Millionen Erwerbslose. Da weder Exportsteigerungen noch eine Erhöhung der Inlandsnachfrage die starken Arbeitsplatzverluste ausgleichen konnten, setzten die Gewerkschaften auf Umverteilung von Arbeit. Den gewerkschaftlichen Vorschlag, Beschäftigung durch ein massives Investitionsprogramm zu fördern, hatte die Bundesregierung ignoriert.

Die Gewerkschaften bekamen zunehmend Legitimationsprobleme, da es ihnen in den Tarifverhandlungen nicht gelang, zumindest den Reallohn zu sichern. Zusätzlich wurden sie durch das 1982 vom Nachrichtenmagazin »Der Spiegel« veröffentlichte Neue-Heimat-Desaster belastet: Missmanagement und Korruption waren in den gewerkschaftseigenen Wohnungs- und Städtebauunternehmen der »Neue-Heimat-Gruppe« jahrelang zumindest fahrlässig geduldet worden. Wie verunsichert insbesondere die gewerkschaftlichen Vertrauensleute waren, ist an der Mitgliederwerbung abzulesen. Wurden 1981 noch 34.000 neue Mitglieder für die GTB geworben, waren es 1982 nur noch 24.000 und ein Jahr später $23.000 .{ }^{52}$ Alle Gewerkschaften brauchten dringend Erfolge.

Der GTB-Gewerkschaftstag 1982 war entschlossen, weitere Schritte der Arbeitszeitverkürzung durchzusetzen, ohne sich auf die Forderung nach der 35-Stunden-Woche festzulegen. Im entsprechenden Antrag wurde betont, dass die »jeweilige Form der Arbeitszeitverkürzung [...] von den Gegebenheiten in den einzelnen Branchen und der Beschäftigungsstruktur « abhänge. ${ }^{53}$ Ende 1982 hatte sich die tarifpolitisch führende IG Metall nach kontroversen Diskussionen auf die Verkürzung der Wochenarbeitszeit festgelegt, die in der Tarifrunde im Frühjahr 1984 in Angriff genommen werden sollte. Auch die IG Druck und Papier bereitete sich darauf vor, die 35-Stunden-Woche durchzusetzen.

Die Unternehmerverbände sprachen sich kategorisch gegen eine weitere Reduzierung der Wochenarbeitszeit aus. In einem sogenannten »Tabu-Katalog« stellte die Bundesvereinigung der Deutschen Arbeitgeberverbände (BDA) schon 1978 fest, dass »eine weitere Reduzierung des Arbeitsvolumens durch Verkürzung der Wochenarbeitszeit unter 40 Stunden keinesfalls zu verantworten« sei. »Sie würde das notwendige wirtschaftliche Wachstum ernstlich beeinträchtigen und damit auch die Wettbewerbsfähigkeit der deutschen Wirtschaft gefährden. ${ }^{54}$

52 GTB: Geschäftsbericht 1982-1985 des Hauptvorstandes, S. 365.

53 GTB: Protokoll des 14. Ordentlichen Gewerkschaftstages, 17.-22. Oktober 1982 in Mainz, S. 381.

54 BDA (1978): Katalog zur Koordinierung der lohn- und tarifpolitischen Fragen, in: Frankfurter Rundschau, Ausgabe vom 26.1.1979, S. 13. 
Wegen höherer Arbeitskosten führe die 35-Stunden-Woche nicht zu einem Rückgang sondern vielmehr zu einem Anstieg der Arbeitslosigkeit.

Dieter Kirchner, Hauptgeschäftsführer von Gesamtmetall, brachte die ablehnende Haltung der Unternehmer zur 35-Stunden-Woche auf die Formel »Lieber vier Wochen Streik als eine Minute Arbeitszeitverkürzung «. ${ }^{55}$ Die Arbeitgeber setzten auf eine Arbeitszeitflexibilisierung und Ausweitung der Produktionszeiten, in der Textilindustrie forderten sie die Möglichkeit zur regelmäßigen Samstagsarbeit. Die Bundesregierung stützte die Arbeitgebersicht und Bundeskanzler Kohl nannte die 35-Stunden-Woche »absurd, dumm und töricht «. ${ }^{56}$

In der Bevölkerung und selbst bei den Gewerkschaftsmitgliedern war die Forderung nach der 35-Stunden-Woche Anfang 1984 heftig umstritten. Je näher die konkrete Auseinandersetzung kam, umso mehr schwand die Zustimmung. Nach allen Umfragen standen auch die meisten Gewerkschaftsmitglieder dieser Forderung skeptisch gegenüber. »Der Spiegel«schrieb:

»Wohl noch nie in der Ceschichte der Metaller-Cewerkschaft stand die Führung so allein mit einer Forderung wie dieses Mal. Noch nie konnten sich die Unternehmer einer so breiten Zustimmung zu ihrem Nein erfreuen wie im Frühjahr 1984. (57 $^{57}$

In der Phase der Zuspitzung über den Einstieg in die 35-Stunden-Woche scherten die IG Chemie, die IG Bau-Steine-Erden, die IG Bergbau und Energie, die Gewerkschaft Nahrung-Genuss-Gaststätten (NGG) und die GTB aus der Riege der Streiter für eine Wochenarbeitszeitverkürzung aus. Sie griffen die verbreitete kritische Stimmung auf und setzten stattdessen auf die populäre Verkürzung der Lebensarbeitszeit. So forderten sie einen Vorruhestand, durch den ein gewisser Prozentsatz von Beschäftigten ab 58 Jahren vorzeitig aus dem Arbeitsleben ausscheiden könne.

Der Charme einer solchen Lösung lag darin, dass die Bundesregierung signalisiert hatte, sich an den Kosten dieser tariflichen Frühverrentung zu beteiligen. Bereits im ersten Quartal 1984 brachte sie den gesetzlichen Rahmen für dieses Modell im Eiltempo auf den Weg. Das Ziel von Arbeitsminister Norbert Blüm und der Bundesregierung war eindeutig: Der Vorruhestand sei Munition im Kampf gegen die 35-Stunden-Woche, wie »Der Spiegel« mit Blick auf Bundesfinanzminister Stoltenberg feststellte. Nach diesem Modell hatte der Arbeitgeber bis zum Rentenbeginn 65 Prozent des früheren Bruttoeinkommens zu zahlen; 35 Prozentpunkte davon sollte er von der Arbeitslosenversicherung erstattet bekommen,

55 Zit. nach Mayr (1984): Der Kampf um die 35-Stunden-Woche, S. 664.

56 Der Spiegel (1984): Arbeitszeit-Noch viel zu mobilisieren, in: Ausgabe 3 vom 16.1.1984, S. 81.

57 Der Spiegel (1984): 35-Stunden-Woche - Zufällig über den Weg, in: Ausgabe 13 vom 26.3.1984, S. 19. 
sofern eine Neueinstellung erfolgte. Eine Aufstockung dieser 65 Prozent sollte über Tarifverträge vereinbart werden können. Das Vorruhestands-Gesetz wurde am 29. März 1984 gegen den heftigen Widerstand der SPD vom Bundestag verabschiedet. In den Branchen, die von den »Vorruhestands-Gewerkschaften« vertreten wurden, fielen 170.000 aufgrund ihres Alters berechtigte Beschäftigte unter das auf vier Jahre befristete Gesetz. ${ }^{58}$

Die GTB wollte nicht die Organisation sein, die den anderen in den Rücken fällt, nur weil Arbeitgeber und Regierung die Lebensarbeitszeitverkürzung favorisierten. In ihrer Mitgliederzeitschrift veröffentlichte sie einen Beitrag des DGBVorsitzenden Ernst Breit mit der Überschrift »Alle Möglichkeiten nutzen «. Dieser schrieb, »die Gewerkschaften haben die Verkürzung der Wochenarbeitszeit mit dem Ziel der 35-Stunden-Woche zu ihrer zentralen Aufgabe erklärt«. Er betonte jedoch, dass man zugleich eine akzeptable Vorruhestandsregelung brauche: »Wir brauchen beides, und zwar dringend. ${ }^{59}$ Allerdings mussten die Gewerkschaften, die mit der Forderung nach einem Vorruhestandstarifvertrag in die Verhandlungen gingen, die Kröte schlucken, dass damit die 40-Stunden-Woche für mehrere Jahre festgeschrieben wurde.

Am 14. Mai 1984 begann der Streik für die Wochenarbeitszeitverkürzung in der baden-württembergischen Metallindustrie. Dieser härteste Streik der Nachkriegsgeschichte, der eine Woche später auf das Tarifgebiet Hessen ausgeweitet wurde, hatte bundesweite Auswirkungen. Gezielt führte die IG Metall strategisch wichtige Zuliefererbetriebe in den Arbeitskampf. Bundesarbeitsminister Blüm wies die Arbeitsämter entgegen der bisher üblichen Praxis an, kein Kurzarbeitergeld an Beschäftigte außerhalb der Streikbezirke zu zahlen, wenn sie wegen eines streikbedingten Materialmangels nicht beschäftigt werden konnten. Dieses Vorgehen stuften die Sozialgerichte aufgrund fehlender gesetzlicher Grundlagen als rechtswidrig ein und kippten den Ministererlass. Die Bundesregierung »rächte« sich noch im selben Jahr mit der Einführung des $\$ 116$ im Arbeitsförderungsgesetz, der die Zahlung von Kurzarbeitergeld für indirekt vom Arbeitskampf Betroffene ausschloss.

Am 28. Juni wurde der Öffentlichkeit ein vom früheren Verteidigungsminister Georg Leber vermittelter Kompromiss vorgestellt. Die IG Metall hatte die Verkürzung der Wochenarbeitszeit auf 38,5 Stunden erreicht, im Gegenzug musste sie zustimmen, dass künftig die Arbeitszeit betrieblich deutlich flexibler gestaltet werden konnte. Durch diesen als »Leber-Kompromiss« bezeichneten Tarifabschluss lag die tarifliche Wochenarbeitszeit in einer bedeutenden Industriebranche erstmals unterhalb der seit fast zwanzig Jahren geltenden 40-Stunden-Woche.

58 Der Spiegel (1984): Rente - Fein Cemacht, in: Ausgabe 11 vom 12.3.1984, S. 28.

59 Breit (1984): Alle Möglichkeiten nutzen, S. 3. 
Während die Metaller streikten, begannen die Verhandlungen für die Textil- und die Bekleidungsbranche. Die Arbeitgeber begrüßten zwar den Weg der Lebensarbeitszeitverkürzung, mauerten jedoch bei den Konditionen. Die von der GTB geforderte tarifliche Aufstockung auf 75 Prozent des vorherigen Einkommens lehnten sie $a b$, außerdem wollten sie sich weitgehende Vetorechte bei Anträgen der Beschäftigten sichern. Die Gewerkschaft rief zu Warnstreiks auf und beschloss Vorbereitungsmaßnahmen für einen Arbeitskampf. Knapp 60.000 Beschäftigte in 335 Textilbetrieben beteiligten sich an Warnstreikaktionen zur Unterstützung der Tarifforderung. ${ }^{60}$ Noch vor den geplanten Urabstimmungen über einen unbefristeten Streik schloss die GTB am 8. Juni für die Textilindustrie im Bezirk Nordrhein einen Tarifvertrag mit einer Vorruhestandsregelung ab und musste im Gegenzug der Unkündbarkeit der 40-Stunden-Woche bis 1988 zustimmen. ${ }^{61}$ Der Tarifvertrag wurde in allen Tarifgebieten der Textilindustrie und in der Bekleidungsindustrie übernommen.

In der Textilindustrie mit ihrer flächendeckenden Schichtarbeit stieß der Vorruhestand auf breite Zustimmung. In der Bekleidungsindustrie profitierten nur wenige davon, da es wegen der Arbeit im Leistungslohn nur wenige ältere Beschäftigte in der Produktion gab. Darüber hinaus konnten sich viele Bekleidungsbeschäftigte die mit dem Vorruhestand verbundenen finanziellen Einkommenseinbußen nicht leisten. Im Gegensatz zu der nach langem Arbeitskampf in der Metall- und Druckindustrie durchgesetzten stufenweisen Verkürzung der Wochenarbeitszeit war der Beschäftigungseffekt der vorgezogenen Altersruhe in den textilen Branchen messbar. Da die Arbeitgeber Zuschüsse zum Vorruhestandsgeld nur erhielten, wenn sie die freiwerdenden Arbeitsplätze neu besetzten, konnte eine positive Beschäftigungswirkung belegt werden. Die vom Institut für Arbeitsmarkt- und Berufsforschung der Bundesanstalt für Arbeit prognostizierte Wirkung für 40.000 bis 70.000 Beschäftigte ${ }^{62}$ wurde mit 80.000 wiederbesetzten Stellen übertroffen. ${ }^{63}$

\subsubsection{Das vierte Welttextilabkommen}

Parallel zur Arbeitszeitverkürzung ging der Kampf zum Schutz vor Importen aus Niedriglohnländern weiter. Das 1982 abgeschlossene und bis Mitte 1986 befristete dritte Welttextilabkommen (WTA; siehe Kapitel 6.1.2) sollte nach Meinung vieler Lieferländer auslaufen. Auch der Verband des europäischen Groß- und Außenhandels und die Verbraucherverbände forderten die Abschaffung der Handels-

60 textil-bekleidung, Ausgabe 7-8/1984, S. 6.

61 GTB: Geschäftsbericht 1982-1985 des Hauptvorstandes, S. 451.

62 IAB (1984): Chronik der Arbeitsmarktpolitik: Vorruhestandsgesetz (VRG).

63 GTB: Geschäftsbericht 1982-1985 des Hauptvorstandes, S. 451. 
beschränkungen. Bereits Ende 1984 wurde auf Initiative der GTB der Europäische Gewerkschaftsausschuss Textil-Bekleidung-Leder (EGV-TBL) aktiv. Um die Zukunft der europäischen Industrien zu retten, dürfe die Importsteigerung die Verbrauchssteigerung in den nächsten Jahren nicht überschreiten, so die Forderung. Im Zentrum stand wieder die Sozialklausel, nach der sich alle Lieferländer verpflichteten, die Kernarbeitsnormen der ILO einzuhalten. Die europäischen Gewerkschaften betonten:

»Die Industrialisierung der Entwicklungsländer muß zu einer echten Verbesserung der Lebensbedingungen der dortigen Bevölkerung und zur Schaffung einer wirklichen Kaufkraft führen. Eine solche Entwicklung würde einen zunehmenden Absatz der Erzeugnisse auf den einheimischen Märkten erlauben. Sie würde aber auch die nationalen Industrien von dem starken Exportzwang und der äußerst scharf gewordenen Konkurrenz befreien, diese zerstört jegliche Aussicht auf einen bedeutsamen sozialen oder wirtschaftlichen Fortschritt. ${ }^{64}$

Gleichzeitig wurde Druck auf Bundeswirtschaftsminister Martin Bangemann und die EG-Kommission aufgebaut. Erstmals gab es eine Anhörung zu dieser Frage im Europäischen Parlament, ${ }^{65}$ bei der der GTB-Vorsitzende Berthold Keller auf die Importquoten hinwies: 76 Prozent aller in der Bundesrepublik verkauften Herrenhosen und 85 Prozent der Herrenmäntel wurden importiert; bei Hemden betrug der Einfuhranteil bereits 93 Prozent. Nach Gesprächen mit der Gewerkschaftsspitze wollte sich der Wirtschaftsminister zwar für eine Verlängerung des WTA einsetzen, diese solle jedoch den Übergang zum Freihandel organisieren. ${ }^{66}$ Keller widersprach vehement.

Zwei Jahre vor dem Auslaufen des bestehenden Abkommens startete die GTB ihre Kampagne für ein neues WTA. Das ganze Jahr 1985 über sollte es Flugblattaktionen, Podiumsgespräche mit Politiker:innen aus textilen Schwerpunktregionen und regionale Kampagnen geben. Mehr als 2.000 Schreiben von Betriebsräten erreichten das Bundesministerium für Wirtschaft, die GTB übergab 170.000 Unterstützungsunterschriften von Beschäftigten und selbst auf dem Jugendempfang des Bundespräsidenten warb die Gewerkschaftsjugend für einen fairen statt freien Welthandel. Bangemann blieb dabei, dass der seit zwölf Jahren geltende Schutz der heimischen Industrien als Übergangszeit ausreichend gewesen sei und die Weichen in Richtung Freihandel gestellt werden müssten.

Damit konnten sich die europäischen Textilgewerkschaften nicht abfinden. Am 21. März 1986 bildeten 4.000 Beschäftigte aus der Textil- und Bekleidungs-

64 Ringelstein (1985): Klartext geredet, S. 14.

65 Ringelstein (1985): Klartext geredet, S. 15.

66 textil-bekleidung, Ausgabe 2/1985, S. $18 \mathrm{f}$. 
industrie Europas eine Menschenkette um die EG-Zentrale und brachten ihre Unzufriedenheit mit den Regierungen der EG-Länder zum Ausdruck. ${ }^{67}$ Der Ministerrat hatte der EG-Kommission ein Verhandlungsmandat mit weitreichenden Zugeständnissen für eine weitere Liberalisierung des Außenhandels erteilt. Die Proteste zeigten Wirkung: Das Europäische Parlament sprach sich gegen einen unbegrenzten Freihandel aus und stellte sich hinter die Gewerkschaftsforderung, kein endgültiges Auslaufdatum für das WTA festzulegen.

Letztlich erzwang jedoch der Druck aus den USA den Abschluss des vierten WTA. Das dortige Repräsentantenhaus hatte den Stopp aller Textil- und Bekleidungseinfuhren aus Entwicklungsländern beschlossen und das Veto des Präsidenten wäre wahrscheinlich überstimmt worden, wenn die Verhandlungen um das globale Abkommen gescheitert wären. ${ }^{68}$ Im Wesentlichen wurde das dritte WTA für weitere fünf Jahre verlängert; die Lieferländer konnten ihre Forderung nach deutlichen Quotenerhöhungen nicht durchsetzen. Eine leichte Verbesserung aus Sicht der Importländer erfolgte dadurch, dass neben Produkten aus Wolle, Baumwolle und Chemiefasern nun auch »sonstige Fasern« wie z. B. Seide einbezogen wurden. Die geforderte Sozialklausel war auch im neuen Abkommen nicht zu finden.

Auf dem Gewerkschaftstag 1986 wurden beide Schwerpunktthemen der vergangenen Jahre bewertet: der tarifliche Vorruhestand und das Ringen um das neue WTA. Der Kurs des Vorrangs einer Lebensarbeitszeitverkürzung wurde nur milde kritisiert. Einzelne Delegierte bemängelten die geringe Wirkung in der Bekleidungsindustrie und die faktische Bevorzugung von Besserverdienern, die auch $\mathrm{zu}$ einer überproportionalen Inanspruchnahme durch Männer geführt habe. ${ }^{69}$ Auf Kritik stieß auch, dass der Schwerpunkt der Gewerkschaftsarbeit stark auf die Handelspolitik gelegt wurde. Einige Delegierte merkten an, dass dies zulasten der Tarifpolitik gegangen sei. ${ }^{70}$

Mit vierzig Wortmeldungen zum Geschäftsbericht des Hauptvorstandes wurde eine lebhafte Debatte um die Ausrichtung der Gewerkschaftspolitik geführt. Nach dreizehn Jahren sozialliberaler Koalition sah man sich mit einer Regierung konfrontiert, die den Gewerkschaften grundsätzlich kritisch gegenüberstand. Die Frage, was Tarifpolitik unter diesen Bedingungen leisten könne, stand im Mittelpunkt der erforderlichen Positionsbestimmung. Die Beschlüsse

67 textil-bekleidung, Ausgabe 4/1986, S. 6f. (»Menschenkette um die EG-Zentrale«).

68 Keller (1986): Unser Einsatz hat sich gelohnt, S. 6 f.

69 So der Delegierte Gerd Will, in: GTB: Protokoll des 15. Ordentlichen Cewerkschaftstages, 5.9. Oktober in Aachen, S. $129 \mathrm{ff}$.

70 So der Delegierte Peter Artzen, in: GTB: Protokoll des 15. Ordentlichen Cewerkschaftstages, 5.-9. Oktober in Aachen, S. $116 \mathrm{f}$. 
des Gewerkschaftstages fokussierten sich auf die Handelspolitik, insbesondere die Zukunft des WTA, und die Kritik an \$116 Arbeitsförderungsgesetz und dem Beschäftigungsförderungsgesetz, das erstmals die sachgrundlose Befristung von Arbeitsverhältnissen ermöglichte. ${ }^{71}$

\subsubsection{Kürzere, aber auch flexiblere Arbeitszeit}

Mit Blick auf die weitere Arbeitszeitpolitik hieß es im Leitantrag des Hauptvorstandes an den Gewerkschaftstag 1986, dass neben Verlängerung und Verbesserung der Vorruhestandstarifverträge "nunmehr alle organisatorischen Kräfte auf eine deutliche Verkürzung der Wochenarbeitszeit zu konzentrieren « seien. ${ }^{72}$ Die schwarz-gelbe Koalition sollte das Vorruhestandsgesetz allerdings nicht über 1988 hinaus verlängern, so dass dieser Tarifregelung der Boden entzogen wurde und die GTB sich 1988 auf den Weg in Richtung 35-Stunden-Woche machte.

Trotz des 1984 in der Metallindustrie erzielten Durchbruchs (siehe Kapitel 6.2.1) erwies sich dies als ein schwieriges Unterfangen, auch wenn sich die wirtschaftliche Situation verbessert hatte: Die Umsätze der Textilindustrie stiegen 1984 und 1985 kräftig, der Arbeitsplatzabbau bei Textil blieb von 1984 bis 1988 durchschnittlich unter 2 Prozent, bei Bekleidung bei 2,5 Prozent pro Jahr. Dennoch bedeutete das in diesen Branchen einen Arbeitsplatzverlust von 50.000 Beschäftigten innerhalb von vier Jahren. ${ }^{73}$ Angesichts von über zwei Millionen Arbeitslosen hatten die Industriegewerkschaften jedoch Schwierigkeiten, die Reallöhne zu verbessern oder gar zu halten. Hinzu kam, dass Tariflohnerhöhungen nicht in vollem Umfang in den Betrieben realisiert werden konnten. Es kam zur sogenannten negativen Lohndrift, d.h. die Reallohnsteigerungen fielen geringer aus als die Tariferhöhungen, weil übertarifliche Einkommensbestandteile gekürzt wurden. ${ }^{74}$

Die Arbeitgeber sahen in der Wochenarbeitszeitverkürzung nichts weiter als eine zusätzliche Kostenbelastung, gegen die sie sich mit aller Kraft stemmten. Sie verlangten flexible Arbeitszeiten, die je nach Auftragslage angepasst werden konnten, sowie den Wegfall der Mehrarbeitszuschläge. Entsprechende Vorschläge brachten sie nun in jeder Tarifrunde als »Gegenforderungen« ein. Unter dem Schlagwort "Deregulierung" sollten auch andere Tarifstandards aufgeweicht werden und z. B. Neueinstellungen unterhalb der tariflichen Mindestlöhne möglich werden. So wurden die Tarifabschlüsse auch nach Überwindung der Wachs-

71 Vgl. GTB: Protokoll des 15. Ordentlichen Gewerkschaftstages, 5.-9. Oktober 1986 in Aachen, S. $401 f f$.

72 GTB: Protokoll des 15. Ordentlichen Cewerkschaftstages, 5.-9. Oktober 1986 in Aachen, S. 410.

73 Statistisches Bundesamt (Destatis) (2008): Fachserie 4, Reihe 4.1.1.

74 CTB: Geschäftsbericht 1986-1989 des Hauptvorstandes, S. 434. 
tumskrise zu Beginn der 1980er Jahre zunehmend zu Abwehrschlachten und gelangten nur nach intensiven Warnstreiks. Immerhin schaffte es die GTB, für die Beschäftigten in der Textil- und der Bekleidungsindustrie in den Jahren 1986 und 1987 wieder bescheidene Reallohnsteigerungen durchzusetzen. ${ }^{75}$

Die Arbeitszeitflexibilisierung war an der Gewerkschaftsbasis äußerst unbeliebt, weil damit Einkommenseinbußen verbunden waren. Insbesondere in der Bekleidungsindustrie gab es bereits stark schwankende Wochenarbeitszeiten, allerdings hatten die Arbeitgeber hierfür Zuschläge in Form von Geld oder zusätzlicher bezahlter Freizeit zu gewähren. Das Problem von am Wochenende stillstehenden teuren Produktionsanlagen war in vielen Textilbetrieben durch regelmäßige Mehrarbeit am Samstag entschärft worden. Käme es zu einem Tarifvertrag, der den ganzen Samstag als Produktionstag ermöglichte, würden durch neue Schichtsysteme zwar neue Arbeitsplätze geschaffen, aber der zusätzlich vergütete Verdienst entfiele für die im bisherigen Schichtsystem Beschäftigten.

Die GTB-Spitze signalisierte ihre grundsätzliche Zustimmung zur Samstagsarbeit, wenn der Sonntag arbeitsfrei bliebe und als Kompensation eine bezahlte Reduzierung der Wochenarbeitszeit von 40 auf 36 Stunden erfolgen würde. Innerhalb der Tarifkommissionen war diese Lösung umstritten, wobei weniger die Belastung durch die Samstagsarbeit als der mögliche Wegfall der Mehrarbeit eine Rolle spielte.

In der Bekleidungsindustrie lag es auf der Hand, dass der Vorteil der Inlandsproduktion umso größer ist, je schneller die Reaktionsmöglichkeiten auf das schwankende Einzelhandelsgeschäft waren. Wenn bestimmte Artikel wetter- oder modebedingt besonders gut liefen, konnten sogenannte "Nachorders" aus deutscher Fertigung noch für die laufende Saison geliefert werden, was bei Produkten aus Fernost war so kurzfristig nicht möglich war. Daher wollten die Arbeitgeber bei hoher Nachfrage alle Möglichkeiten der damals geltenden gesetzlichen Arbeitszeitordnung (AZO) ausschöpfen, was bis zu 60 Arbeitsstunden pro Woche bedeutet hätte. Möglichst viel davon sollte dann »vorgearbeitete Zeit«sein, die zu nachfrageschwachen Zeiten wie dem Saisonwechsel »abgefeiert« würde.

Die Unternehmen setzten auf schnelle Reaktionsfähigkeit und niedrigere Kosten. Darüber hinaus hatte eine durch flexible Arbeitszeit kürzere Produktionsperiode den Vorteil, dass Stoffe später bestellt werden konnten. So war weniger Kapital im Produktionsprozess gebunden, weil die Vorfinanzierung der Ware für einen kürzeren Zeitraum fällig war.

Viele Betriebe nutzten schon in den 1960er und 1970er Jahren eine Bestimmung in der AZO, nach der Vor- und Nacharbeit zuschlagsfrei für Freizeiten »im Zusammenhang mit Feiertagen und Volksfesten « innerhalb eines Zeitraums von 7 Wochen möglich war. Im 1979 vereinbarten neuen Manteltarifvertrag für 
die Bekleidungsindustrie hatte die GTB diese Bestimmung in den Vertrag aufgenommen und erweitert. Die neue Regelung ermöglichte die Verlängerung der Wochenarbeitszeit auf bis zu 45 Stunden, wenn sie mit einem Zuschlag von 25 Prozent in Form von Geld oder Freizeit gewährt wurde. Der Freizeitausgleich musste innerhalb von 13 Wochen erfolgen. Somit konnte für 32 »vorgearbeitete« Stunden eine ganze 40-Stunden-Woche »freigenommen « werden. Zugleich waren der Flexibilisierung auch Grenzen gesetzt: Die tägliche Arbeitszeit war per Tarifvertrag auf 9 Stunden beschränkt, der Samstag sollte grundsätzlich frei bleiben.

Trotz der Belastung durch längere Arbeitszeiten waren die dadurch möglichen Freizeitblöcke bei vielen Beschäftigten beliebt. In den meisten größeren Betrieben der Bekleidungsindustrie wurden entsprechende Betriebsvereinbarungen abgeschlossen. In den Tarifverhandlungen stellten die Arbeitgeber die Zuschläge zunehmend in Frage und wollten den 13-Wochen-Zeitraum ausweiten.

Die GTB verband ihre Bereitschaft, den Arbeitgebern der Textilindustrie bei der Samstagsarbeit entgegenzukommen, mit der Forderung nach deutlich verbesserten Rationalisierungsschutzabkommen. Dadurch sollten Betriebsräte Möglichkeiten erhalten, die technologische Entwicklung mitzugestalten. Zugleich sollte ein solcher Tarifvertrag die Beschäftigten vor Einkommens- oder Arbeitsplatzverlust schützen. Die Ansätze zum Rationalisierungsschutz aus den 1960er Jahren wurden weiterentwickelt und sollten nun flächendeckend vereinbart werden. Dies passte zum Ansatz der Beschäftigungssicherung, der auch Hauptargument für die geforderte Wochenarbeitszeitverkürzung war. In der Tarifrunde 1988 stand nun das ganze Paket auf der Agenda; 35-Stunden-Woche und Rationalisierungsschutz wurden als Instrumente zur Arbeitsplatzsicherung herausgestellt. Mit diesem Argument machte die GTB auch auf der politischen Ebene in der Außenhandelspolitik Druck.

Insbesondere in der Bekleidungsindustrie genoss die Wochenarbeitszeitverkürzung eine hohe Popularität und ein großes Mobilisierungspotenzial. Der Geschäftsbericht bezeichnet die Tarifrunde 1988 rückblickend als die längste und schwierigste der Nachkriegszeit. ${ }^{76}$ Erstmals setzte die GTB hier das Instrument des Halbtagesstreiks ein. An einem Freitag wurde zu einem vierstündigen Warnstreik aufgerufen, die GTB-Mitglieder erhielten eine pauschale Streikunterstützung. Insgesamt beteiligten sich über 110.000 Beschäftigte an den Warnstreiks, davon stellte die Bekleidungsindustrie mit 60.000 Streikenden erstmals mehr als die Hälfte. Die Tarifverträge waren zum 30. April gekündigt worden, aber erst in der zehnten Verhandlungsrunde wurde im Tarifbezirk Textilindustrie Nordrhein am 7. Juni 1988 der Durchbruch erzielt. In der Bekleidungsindustrie musste der Druck weiter erhöht werden, bis das Ergebnis am 5. Juli 1988 unterzeichnet werden konnte.

76 CTB: Ceschäftsbericht 1986-1989 des Hauptvorstandes, S. 446. 
Der Tarifabschluss brachte das Ende der seit siebzehn Jahren geltenden 40-Stunden-Woche. Für das Folgejahr sah er zunächst eine Stunde und im darauffolgenden Jahr eine weitere halbe Stunde Arbeitszeitverkürzung vor. Gleichzeitig gelang es, ein Rationalisierungsschutzabkommen zu vereinbaren, auf das in Kapitel 6.3.2 genauer eingegangen wird.

Für die Textilindustrie mit Ausnahme Südbayerns wurden Tarifverträge über die »Optimierung der Maschinenlaufzeiten« abgeschlossen. Auf der Grundlage von nicht erzwingbaren Betriebsvereinbarungen konnte der Samstag in die Verteilung der regelmäßigen Wochenarbeitszeit aufgenommen werden. Wirksam wurde die Vereinbarung allerdings erst mit Zustimmung der Tarifvertragsparteien. Für die Samstagsarbeit wurden Zuschläge fällig, die bei Einbezug des ganzen Samstages einen bezahlten Freizeitausgleich von drei Stunden wöchentlich ermöglichten oder in Geld abgegolten wurden.

Die GTB, die es seit fast zwanzig Jahren mit einer stetig schrumpfenden Textil- und Bekleidungsindustrie zu tun hatte, bewies mit den umfangreichen Tarifabschlüssen 1988 erneut ihre Gestaltungskraft und hatte im Vergleich zu anderen Branchen gute Ergebnisse durchgesetzt. Bei der tariflichen Arbeitszeitflexibilisierung lief sie nicht der Entwicklung in den Betrieben hinterher, sondern schaffte erweiterte Rahmenbedingungen für die betriebliche Gestaltung. Zwar standen damit die Betriebsräte unter Druck, entsprechende FlexibilisierungsVereinbarungen zu treffen, aber die Tarifverträge sicherten Gegenleistungen wie die Arbeitszeitverkürzung bei regelmäßiger Samstagsarbeit ab. Der Organisationsgrad war nach 1981 von 50,8 Prozent auf 46,3 Prozent im Jahr 1986 gesunken, was sowohl auf das Neue-Heimat-Desaster (siehe Kapitel 6.2.1) als auch auf die tiefgreifende Branchenkrise bei Textil und Bekleidung zurückzuführen ist. Durch die hohe Mobilisierung für die Tarifrunde 1988 wurde dieser Trend gedreht und der Organisationsgrad verbesserte sich wieder auf 47,6 Prozent. Trotz weiter sinkender Beschäftigung wurden bis Ende der 1980er Jahre jährlich rund 25.000 neue Mitglieder geworben. ${ }^{77}$

Der Preis, der für die Tarifabschlüsse des Jahres 1988 gezahlt werden musste, war zum einen die sehr lange Mindestlaufzeit der Tarifverträge über Lohn und Gehalt von drei Jahren, zum anderen fielen die Einkommenserhöhungen für die Jahre mit Arbeitszeitverkürzung trotz vollem Lohnausgleich, der zusätzlich erfolgte, sehr bescheiden aus, nämlich 1,2 Prozent für 1989 und 2,o Prozent für 1990. Dies sollte aufgrund des nicht abzusehenden Wirtschaftsbooms der Folgejahre noch für Schwierigkeiten sorgen (siehe Kapitel 6.1.5).

77 GTB: Geschäftsbericht 1986-1989 des Hauptvorstandes, S. 376. 


\subsection{Arbeit gestalten, Arbeitsplätze sichern}

Die Gestaltung der Arbeit ist entscheidend für Gesundheit und Wohlbefinden der Arbeitenden. Doch schon in der Frühzeit der Industrialisierung richtete sich der gewerkschaftliche Schwerpunkt auf die sozialpolitische Folgenabfederung wie Verteilungskampf und Begrenzung der Arbeitszeit. Die Fragen der Arbeitsgestaltung und -organisation standen nicht im Fokus. Die Vorstellungen der »romantischen Frühsozialisten« Robert Owen und Charles Fourier über die humane Gestaltung der Arbeit wurden von den »wissenschaftlichen Sozialisten« nicht aufgenommen, so dass sich keine sozialistische Theorie der Arbeitsgestaltung entwickelte. $^{78}$

Unzweifelhaft war die Bekämpfung der nicht existenzsichernden Einkommen zu Beginn der Industrialisierung und in der langen Folgezeit die Kernaufgabe der Arbeiterbewegung. Erst im nächsten Schritt wurde die Forderung nach angemessenem Arbeitsschutz entwickelt. Hier sah man den Staat in der Pflicht, der dieses Feld erst nach der Revolution 1918 und Gründung der Weimarer Republik in Angriff nahm. Die Gewerkschaften drängten auf ein konsequenteres Handeln, stellten jedoch nicht die Frage, wie die Beschäftigten selbst auf die technische Entwicklung Einfluss nehmen können. Im Vordergrund stand das Verständnis von Rationalisierung als Chance, um die Produktivität zu steigern und dadurch Arbeitszeitverkürzungen und Lohnerhöhungen finanzieren $\mathrm{zu}$ können. ${ }^{79}$

Angestoßen durch die Reformpolitik der sozialliberalen Koalition setzte in den 1970er Jahren ein Umdenken ein, das auch die GTB erfasste. Beim Ringen um bessere Arbeitsgestaltung erzielte sie beachtliche Erfolge und ging mit einem Tarifvertragsentwurf zur sozialen Gestaltung von Rationalisierung deutlich über die traditionelle Sicht hinaus, nämlich die soziale Folgenabmilderung von Rationalisierungsmaßnahmen. Vielmehr stellte die GTB den Anspruch, die Eingriffsrechte von Betriebsräten per Tarifvertrag deutlich zu erweitern und den Schutz der Beschäftigten in allen Fällen, wo menschliche Arbeit durch Technik ersetzt wird, integrativ in die Entscheidungsprozesse zu verankern.

\subsubsection{Humanisierung der Arbeit - von Lärm bis Licht}

Ende der 1950er Jahre hatte die Textilindustrie die Mechanisierung und Automatisierung der Produktion forciert. Die technologische Entwicklung führte zumindest teilweise zum Abbau körperlicher Belastungen. Zugleich wurden Arbeitsinhalte reduziert und Überwachungstätigkeiten nahmen $\mathrm{zu}$, was vielfach

78 Vgl. Wassermann (1985): Arbeitsgestaltung als Gegenstand gewerkschaftlicher Politik, S. 23 f.

79 Wassermann (1985): Arbeitsgestaltung als Gegenstand gewerkschaftlicher Politik, S. 32. 
zu nervlichen Belastungen führte. ${ }^{80}$ Die Arbeitsplatzumgebung war durch Lärm, Vibration, Staub und teilweise schlechte Lichtverhältnisse geprägt. ${ }^{81}$ In der Bekleidungsindustrie waren schlechte Sitzgelegenheiten, nicht höhenverstellbare Nähmaschinentische, Staub und schlechte Lichtverhältnisse sowie Hitze in den Bügeleien an der Tagesordnung. Anders als in der Textilindustrie waren technische Rationalisierungsmöglichkeiten hier begrenzt - die sogenannte "Biegeschlaffheit« der verarbeiteten Materialien setzt der Automatisierung in der Bekleidungsindustrie bis heute Grenzen.

Die Arbeitsbedingungen waren immer wieder Thema auf Gewerkschaftstagen, ohne dass die Diskussionen zu konkretem Handeln geführt hätten. Das änderte sich 1974, als der GTB-Gewerkschaftstag beschloss, neben den Themen Bezahlung und Beschäftigung auch das Thema Humanisierung des Arbeitslebens (HdA) starkzumachen. Gesundheit und menschliches Wohlbefinden müssten Vorrang vor ökonomischen und technischen Zielen haben, weshalb der Gewerkschaftstag forderte,

»daß in zukünftigen Tarifverträgen Regelungen und Normen über die menschengerechte Gestaltung von Arbeitsplätzen und Arbeitsabläufen als Mindestvoraussetzungen für jegliche Arbeit im Betrieb vereinbart werden ${ }^{82}$

Die GTB legte auch diesen Themenschwerpunkt beteiligungsorientiert an. Durch wissenschaftliche Forschungsprogramme wurden Erkenntnisse gewonnen und entsprechende Umsetzungsmaßnahmen in Pilotbetrieben eingeleitet. Weil die betriebliche Implementierung durch öffentliche Mittel finanziert wurde, gelang es, Unternehmen dafür zu gewinnen. Die GTB bot Seminare von der örtlichen bis zur zentralen Ebene zum Thema Arbeitsgestaltung an, was auch in der Mitgliederzeitung regelmäßig und ausführlich behandelt wurde ${ }^{83}$ Betriebsräte wurden für das Thema der menschengerechten Arbeitsgestaltung sensibilisiert und die Verbesserungen waren für die Beschäftigten erlebbar. In der Textilindustrie gelang es in vielen Betrieben, spürbare Erleichterungen der Arbeitssituation zu erreichen: bessere Beleuchtung, Abtrennung besonders lärmintensiver Maschinen durch nachträglich eingezogene Wände, Absauganlagen und Veränderungen bei der Anordnung von Maschinen und in der Raumaufteilung. ${ }^{84}$

80 Wassermann (1985): Arbeitsgestaltung als Cegenstand gewerkschaftlicher Politik, S. 119.

81 Wassermann (1985): Arbeitsgestaltung als Gegenstand gewerkschaftlicher Politik, S. $129 \mathrm{ff}$.

82 GTB: Protokoll des 12. Ordentlichen Cewerkschaftstages, 3.-8. November 1974 in München, S. 323.

83 CTB: Geschäftsbericht 1974-1977 des Hauptvorstandes, S. 520ff.

84 GTB: Geschäftsbericht 1974-1977 des Hauptvorstandes, S. 255. 
Der 1974 formulierte Anspruch, die Voraussetzungen für bessere Arbeitsgestaltung in Tarifverträgen zu verankern, konnte 1979 in der Bekleidungsindustrie umgesetzt werden. Der Manteltarifvertrag, der neunzehn Jahre nach der Kündigung seines Vorläufers mit der Lohntarifrunde durchgesetzt wurde, brachte nicht nur den sechswöchigen Erholungsurlaub, die Einführung bezahlter Kurzpausen, garantierte Zuschläge für persönliche Bedürfnisse und Erholungsphasen für im Leistungslohn Beschäftigte. Die GTB setzte mit der Vereinbarung auch erweiterte Mitwirkungs- und Mitbestimmungsmöglichkeiten bei der Arbeitsplatzgestaltung für Betriebsräte durch. Damit legte dieser Tarifvertrag die Grundlage für eine Humanisierungswelle.

Nach Inkrafttreten des neuen Manteltarifvertrags startete die GTB die »Aktion bessere Arbeitsplätze«, die sie mitgliedernah durchführte. Die Beschäftigten erhielten Fragebögen zu ihrer konkreten Arbeitssituation, die tausendfach ausgefüllt wurden..$^{85}$ Die Auswertung der Ergebnisse bildete den Auftakt für die Veränderung der Arbeitssituation vieler in der Bekleidungsindustrie Beschäftigter. In den meisten Nähsälen wurden die bislang vorherrschenden schlichten Küchenstühle durch ergonomische Arbeitsstühle ersetzt und die bislang oft unzureichende Beleuchtung verbessert. Außerdem setzten die Betriebsräte in Bekleidungsbetrieben mit Hilfe des Tarifvertrags Absauganlagen an den Bügelarbeitsplätzen mit Hitze- und Dampfentwicklung durch, wie die Mitgliederzeitung in mehreren Ausgaben berichtete. ${ }^{86}$ Mit dieser Aktion bearbeitete die GTB das Thema Arbeitsplatzgestaltung erstmals umfassend.

Das von der Regierung angestoßene und geförderte HdA-Projekt konzentrierte sich auf wenige Pilotbetriebe und zielte auf in die Tiefe gehende arbeitsorganisatorische Veränderungen. Nach Ablauf der geförderten Phase scheiterten diese Ansätze an Betriebsleitungen und Beschäftigten, die sich vielfach durch Gruppenarbeit oder wechselnde Arbeitsaufgaben überfordert sahen. Mit der »Aktion bessere Arbeitsplätze« erreichte die GTB jedoch eine große Breitenwirkung. Die Verbesserungen waren spürbar und erleichterten den Arbeitsalltag. Die Beschäftigten brachten sie mit ihrer Gewerkschaft in Verbindung, die auch für bestehende Arbeitsplätze Mitbestimmungsrechte durchgesetzt hatte und dies offensiv kommunizierte. Die stärkere organisatorische Erschließung der Bekleidungsindustrie war also mit konkreten Verbesserungen über Entgelterhöhungen hinaus verbunden. Für die Steigerung des gewerkschaftlichen Organisationsgrades war die »Aktion bessere Arbeitsplätze« ein maßgeblicher Eckpfeiler.

85 GTB: Geschäftsbericht 1978-1981 des Hauptvorstandes, S. 532.

86 Vgl. textil-bekleidung, Ausgabe 1/1980, S. 9; Ausgabe 6-7/1980, S. 22; Ausgabe 1/1981, S. 15; Ausgabe $7-8 / 1982$, S. $22 \mathrm{f}$. 


\subsubsection{Rationalisierungsschutz - alte Forderungen, neue Erfolge}

Nach den Ergebnissen bei der Gestaltung der Arbeit widmete sich die GTB einem Thema, das ein Vierteljahrhundert zuvor schon einmal Schwerpunkt gewesen war. Karl Buschmann wollte bereits Ende der 1950er Jahre die sozialen Folgen von Rationalisierungen tarifpolitisch abfedern, allerdings ging es damals weder um Einfluss auf die technologische Entwicklung noch um Verlangsamung oder gar einen Stopp der Maßnahmen. In diesem Sinne formulierte Buschmann 1963:

»Nicht die Verhinderung von Rationalisierungsmaßnahmen, nicht die Konservierung der Arbeitsplätze, sondern soziale Sicherheit für unsere älteren Kolleginnen und Kollegen ist das Ziel. ${ }^{87}$

Seitdem konnte die GTB Erfolge mit sogenannten Rationalisierungsschutz-Tarifverträgen verzeichnen, mit denen sie Kündigungsfristen verlängerte und Entlassungen oder Lohnminderungen abfederte, allerdings nur in geringem Umfang. 1985 nahm sich die GTB vor, umfassendere Rationalisierungsschutz-Tarifverträge anzustreben, die weit über den Schutz älterer Beschäftigter hinausgehen. Die grundsätzliche Sichtweise, Rationalisierung als Baustein der Produktivitätssteigerung und damit als Grundlage für höhere Einkommen und bessere materielle Arbeitsbedingungen zu betrachten, änderte sich nicht. Hermann Schumacher, der Tarifverantwortliche im GHV schrieb:

»Nicht die Techniken stehen unserer Forderung nach einer menschenwürdigen Arbeitswelt entgegen, sondern diejenigen, die die Technik für ihre einseitigen Ziele missbrauchen. [...] Dennoch liegt die Lösung des Problems sicherlich nicht in einer Strategie der gewollten Produktivitätsdrosselung oder in einer Neuauflage veralteter Modelle des Maschinensturms. $\ll^{88}$

Auch wenn diese Aussage nach der Position aus den frühen 1960er Jahren klingt, so hatte die Gewerkschaft ihre Konzepte im Laufe der letzten 25 Jahre doch weiterentwickelt. Sie forderte einen Rationalisierungsschutzvertrag, der beim Einsatz neuer Technologien und der daraus folgenden Personalplanung umfassendere Gestaltungsoptionen für Betriebsräte vorsah, sowie ein Recht auf Qualifizierung, berufliche Weiterbildung und Umschulung. ${ }^{89}$

87 CTB: Protokoll des 8. Ordentlichen Cewerkschaftstages, 16.-20. September 1963 in Hannover, S. 67.

88 Schumacher (1987): Mehr Kaufkraft-mehr Schutz, S. 7

89 Schumacher (1987): Mehr Kaufkraft-mehr Schutz, S. 7. 
In der Tarifrunde 1986 brachte die GTB ihre Forderungen ein, und zwar im Gegensatz zu 1963 nicht nur für die Textil-, sondern erstmals auch für die Bekleidungsindustrie. Der Fokus lag auf dem Schutz vor den Folgen von Rationalisierung, Automatisierung und dem Einsatz neuer Technologien, auch im Angestelltenbereich. Die Arbeitgeber blockten und wollten unter allen Umständen Ansprüche der Arbeitnehmervertretungen bei der Einführung neuer Technologien vermeiden, die ihr Alleinentscheidungsrecht auch nur berührten. Die GTB erreichte bei den Tarifverhandlungen 1986 keine Einigung, aber zumindest die Zustimmung von Gesamttextil, weitere Gespräche zu diesem Thema zu führen. Diese Zusage wurde im Folgejahr so konkretisiert, dass man sich wechselseitig verpflichtete, bis zum Jahresende $1987 \mathrm{zu}$ einer Vereinbarung zu kommen. 1988 sollte eine Einigung erzielt sein, denn dann stand die Wochenarbeitszeitverkürzung auf der tarifpolitischen Agenda.

Die Arbeitgeber verhandelten in dieser Frage destruktiv; in der Bekleidungsindustrie zweifelten sie die Notwendigkeit einer tariflichen Regelung grundsätzlich an. ${ }^{90}$ Monatelang wurde in den Verhandlungen ausschließlich über Definitionen gestritten. Dennoch gelang es in der folgenden Tarifrunde 1988, sowohl die 40-Stunden-Woche zu unterschreiten als auch Rationalisierungsschutzverträge für die Textil- und die Bekleidungsindustrie durchzusetzen, die über die Regelungen der 1960er Jahre hinausgingen. Schon wegen des Streitthemas Wochenarbeitszeitverkürzung führte die Tarifrunde $\mathrm{zu}$ einer überdurchschnittlichen Mobilisierung (siehe Kapitel 6.2.3). Die GTB konnte den Arbeitgeberverbänden vermitteln, dass diese Tarifrunde nur mit einem Paket abgeschlossen werden könne, das neben Lohnerhöhung und Arbeitszeitverkürzung auch den Rationalisierungsschutz beinhalten müsse.

Die für die Textil- und die Bekleidungsindustrie bis heute geltenden Rationalisierungsschutz-Tarifverträge beziehen sich auf Maßnahmen, bei denen »Arbeit durch Kapital ersetzt« wird, also auf Neuanschaffung oder Veränderung technischer Anlagen und damit verbundener Umorganisierung von Arbeitsabläufen. Bereits im Planungsstadium sind Betriebsräte umfassend mit einzubeziehen. Vor etwaigen Entlassungen müssen Maßnahmen wie Verzicht auf Neueinstellung und Mehrarbeit, die Einführung von Kurzarbeit, Versetzungen, die gegebenenfalls Umschulungen erfordern, und vorzeitige Verrentung geprüft werden. Im Falle von Versetzung und Abgruppierung, aber auch bei Beendigungskündigung gelten verlängerte Kündigungsfristen. Bei Einkommensminderung werden Ausgleichszahlungen für einen Zeitraum von bis zu sechs Jahren fällig; für entlassene Beschäftigte mit einer Betriebszugehörigkeit von mindestens zehn Jahren

90 So wurde die Verhandlungsführung auch in den Schlussverhandlungen noch angewiesen, einen solchen Tarifvertrag zu verhindern; vgl. BBI: Protokoll der Mitgliederversammlung vom 10. Mai 1988, BWA V11 10. 
sieht der Tarifvertrag Abfindungen vor. Bei der Gestaltung von veränderten oder neu geschaffenen Arbeitsplätzen sind die Grundsätze der menschengerechten Arbeitsgestaltung einzuhalten, wobei die Betriebsräte ein erweitertes Mitbestimmungsrecht haben. ${ }^{91}$

In einem entscheidenden Punkt setzten sich allerdings die Arbeitgeber durch: Ausgliederung von Produktion oder Teilen davon sowie Fälle, in denen ein Sozialplan vereinbart wird, werden von den Tarifverträgen nicht erfasst. Gleiches gilt für Veränderungen, die konjunkturell, saisonal, produkt- oder modebedingt sind. ${ }^{22}$ Dadurch ist der tarifvertragliche Rationalisierungsschutz in der Praxis eher bedeutungslos geblieben. In den ersten Jahren der Gültigkeit spielten die Ausschlusstatbestände nämlich kaum eine Rolle, weil die Branchen zur Zeit des Tarifabschlusses eine lange nicht gekannte Erholung erlebten und der Arbeitsplatzabbau nur gering war.

Die GTB feierte ihren Erfolg: Die Tarifverträge waren für die Textil- und die Bekleidungsindustrie durchgesetzt worden, sie galten erstmals nicht nur für gewerbliche, sondern für alle Beschäftigten. Mit ihrer Forderung hatte die Gewerkschaft den Anspruch auf sozialen Schutz der Beschäftigten bei Textil und Bekleidung deutlich formuliert. Sie hatte dafür gestritten, die Beschäftigten im technischen und im Verwaltungsbereich vor Arbeitsplatz- und Einkommensverlusten insbesondere durch den Einsatz neuer Technologien zu schützen. "Damit nicht noch mehr fallen« hatte die Gewerkschaft ihre Kampagne zur Durchsetzung der Tarifverträge betitelt.

So reihte sich der Kampf um die Durchsetzung des Rationalisierungsschutzes in die anderen Maßnahmen gegen Arbeitsplatzabbau ein. Das Ringen um die Welttextilabkommen, die Durchsetzung des Vorruhestandes, die Wochenarbeitszeitverkürzung und die Lohnerhöhungen zur Stabilisierung der Kaufkraft dienten ebenso der Beschäftigungssicherung wie die neuen RationalisierungsschutzTarifverträge. Mit dieser Themenvielfalt machte die GTB deutlich, dass sie sich mit aller Kraft für die Sicherheit der Arbeitsplätze in ihren Branchen einsetzte. Dass das Ergebnis weit hinter den Zielen zurückblieb, wurde nicht diskutiert.

\subsection{Berthold Keller - Kämpfer für die Arbeitsplätze}

Während der Zeit, in der Karl Buschmann den GTB-Vorsitz innehatte, wurde durch den gesellschaftspolitischen Umbruch ab 1968 und die sozialliberale Koalition ein Reformschub befördert. In der Amtszeit seines Nachfolgers Berthold

91 textil-bekleidung, Ausgabe 7-8/1988, S. $6 \mathrm{f}$.

92 Siehe §3 der Rationalisierungsschutz-Tarifverträge für die Textil- und Bekleidungsindustrie von 1988. 
Keller, der sein Amt Ende 1978 antrat, änderten sich die Rahmenbedingungen für gewerkschaftliche Erfolge deutlich. Die Folgen der Wirtschaftskrise 1978 wurden dadurch gemildert, dass die Regierung Schmidt/Genscher in der zweiten Hälfte der 1970er Jahre Investitionsprogramme auf den Weg gebracht hatte. Als diese ausliefen, verschlechterte sich 1980 die wirtschaftliche Lage spürbar. Nun machte die Opposition die Staatsverschuldung zum Thema. Die FDP drängte innerhalb der Koalition auf einen neoliberaleren Kurs, was schließlich zum Sturz von Bundeskanzler Helmut Schmidt 1982 führte. Die neue schwarz-gelbe Bundesregierung stand für Entstaatlichung, Flexibilisierung und Deregulierung. Die Arbeitslosenunterstützung wurde gekürzt, die Ausbildungsförderung gestrichen, in der Krankenversicherung wurden Eigenbeteiligungen eingeführt. Die Arbeitgeberseite sah endlich wieder eine wirtschaftsfreundlichere Regierung am Ruder.

Die politische Landschaft wandelte sich: In den 1970er Jahren spielten systemkritische Diskussionen eine große Rolle. ${ }^{93}$ Zum Ende des Jahrzehnts wurden Umweltschutzthemen zunehmend relevant, außerdem engagierten sich immer mehr Menschen gegen die geplante Aufstellung neuer Mittelstrecken-Atomraketen in Europa. Beide Themen führten zur Parteigründung der »Grünen«, durch die sich die politische Landschaft in den Folgejahren neu sortierte. In der SPD entbrannte ein Streit um den richtigen Kurs. Der SPD-Vorsitzende Willy Brandt wollte seine Partei für die kritischen Umwelt- und Friedensaktivisten öffnen; der Politologe Richard Löwenthal wandte sich Ende 1981 mit einem Papier gegen diesen Kurs. Er wirkte als rechtes Korrektiv zum "Linken« Erhard Eppler in der SPD-Grundwertekommission mit. In den »Sechs Thesen zur Identität der Sozialdemokratie« spitzte Löwenthal die Diskussion über den Kurs der Partei auf eine »Streitfrage« $\mathrm{zu}$ : Entweder richte sich die SPD

»nach dem Primat der Lebensfähigkeit unserer Industriegesellschaft und der maximalen Beschäftigung ihrer Mitglieder einerseits oder dem Primat nichtindustrieller Lebensformen und der absoluten Verhinderung ökologischer Schäden andererseits ${ }^{94}$

Berthold Keller sah die SPD ebenfalls auf einen postindustriellen Kurs einschwenken und exponierte sich als Befürworter der Löwenthal-Thesen. Dabei ging er direkt Willy Brandt an, nachdem dieser sich ironisch über die »Textilarbeiterin" Annemarie Renger geäußert hatte, die eine Unterschriftenaktion zur Unterstützung der Löwenthal-Thesen initiiert hatte. Neben diesen gesellschaftspolitischen

93 In den Augen der GTB reichte das als gefährlich einzustufende Spektrum von dogmatischen Linken in der SPD wie der Stamokap-Cruppe über die orthodoxen Kommunisten bis hin zu den Maoisten.

94 Zit. nach: Der Spiegel (1981): SPD - Etwas dünn, in: Ausgabe 50 vom 7.12.1981, S. 22. 
Einflüssen von außen gab es ein innergewerkschaftliches Erdbeben: Die Gewerkschaften verloren 1982 durch den vom Nachrichtenmagazin »Der Spiegel« aufgedeckten Skandal um den gemeinwirtschaftlichen Wohnungsbaukonzern »Neue Heimat« enorm an Ansehen. ${ }^{95}$

Keller wollte die GTB auch in dem politisch veränderten Umfeld auf sozialpartnerschaftlichem Kurs halten. Mit seinem Konzept einer veränderten Lohnpolitik in der Krise war er gescheitert (siehe Kapitel 6.1.4); die »Traditionalisten" hatten in der Tarifpolitik eine starke Position. Auch zwanzig Jahre nach der politischen Wende in der GTB wurde keine Tarifrunde ohne Warnstreiks, mehrfach sogar mit Urabstimmungen und befristeten Streiks abgeschlossen. Sehr selbstbewusst und eigenständig trat der Bezirk Nordrhein in der Tarifpolitik auf. Dem grundsätzlich sozialpartnerschaftlichen Ansatz standen immer noch die Bezirke Frankfurt (Hessen, Rheinland-Pfalz, Saarland) und Minden-Lippe (Ostwestfalen) sowie Teile Münster-Arnsbergs (Westliches Westfalen) kritisch gegenüber. Nach dem gescheiterten Vorstoß in Sachen Investivlohn unternahm Keller keine Initiativen mehr, die von diesen Bezirken forcierte aktive Tarifpolitik in irgendeiner Form zu bremsen.

Bis zum Schluss von Berthold Kellers Amtszeit führte die GTB harte TarifAuseinandersetzungen, auch im Ringen um die Wochenarbeitszeitverkürzung und in der Nachschlags-Tarifrunde 1990 (siehe Kapitel 6.1.5). Das Feld der Tarifpolitik hatte Keller weitgehend den Bezirksleitern überlassen, im Zentrum seiner Politik standen die Kampagnen für die Verlängerung und Verbesserung der Welttextilabkommen (siehe Kapitel 6.1.2 und 6.1.3). Als sich Mitte der 1980er Jahre die gesamtwirtschaftliche Entwicklung wieder besserte, konnte der Verlagerungsprozess in den Bereichen Textil und Bekleidung durch die Verbesserung der Handelsbeschränkungsabkommen verlangsamt werden. Noch stärker als unter der Führung Karl Buschmanns verpflichtete Keller bei diesem Thema die Organisation auf eine breite Beteiligung der Mitglieder.

Berthold Keller positionierte sich als Kämpfer für den Erhalt der Arbeitsplätze. Mit dem Ringen um Arbeitszeitverkürzungen und Außenhandelsschutz unterstrich er, dass die GTB sich nicht mit der Opferrolle begnügen werde. Mit seinem Ringen um den Erhalt der europäischen Textil- und Bekleidungsindustrie förderte Keller die Zusammenarbeit der Textil- und Bekleidungs-Gewerkschaften in Europa, deren Höhepunkt eine europaweite Arbeitsniederlegung am 2. Dezember 1980 war (siehe Kapitel 6.1.3). In der Auseinandersetzung um den ersten Schritt der Arbeitszeitverkürzung blieb er an der Seite der Vorruhestandsbefürworter und ermöglichte mehreren tausend Beschäftigten den Ausstieg aus dem Arbeitsleben mit 58 Jahren (siehe Kapitel 6.2.1). 
Als GTB-Vorsitzender vertiefte Berthold Keller auch die Zusammenarbeit mit der Gewerkschaft Nahrung-Genuss-Gaststätten (NGG). Mit Günter Döding, der ebenfalls 1978 zum NGG-Vorsitzenden gewählt worden war, verband ihn das Bestreben, auch den kleinen Gewerkschaften eine starke Stimme im DGB und in der Öffentlichkeit zu geben. Im November 1978 schlossen GTB und NGG auch formell einen Kooperationsvertrag. ${ }^{96}$ Darin verpflichteten sie sich zu gemeinsamem Vorgehen und gegenseitiger Unterstützung bei der Interessenvertretung innerhalb des DGB und gegenüber Regierungen und Parteien. Die gegenseitige Unterstützung sollte alle gewerkschaftlichen Ebenen umfassen und die organisatorische Zusammenarbeit reichte von Publikationen über Schulungen für ehrenamtliche und hauptamtliche Aktive bis hin zur Entwicklung gemeinsamen Werbematerials.

Ein enges Verhältnis hatte der GTB-Vorsitzende zur IG Chemie-Papier-Keramik, der Sprecherin der gemäßigten Gewerkschaften. Diese fuhr in den 1980er Jahren unter ihrem Vorsitzenden, dem konservativen Sozialdemokraten Hermann Rappe, einen streng antikommunistischen und sehr sozialpartnerschaftlich-wirtschaftsfreundlichen Kurs. Während die IG Chemie-Führung jedoch zur Verteidigung dieses Kurses Andersdenkende in ihrer Organisation konsequent isolierte oder sogar ausschloss, ${ }^{97}$ wurden die Kritiker:innen in der GTB als "Stachel im Fleisch« der Gewerkschaft geduldet. Einige der im Zuge der »Vorwärtsstrategie« der 1970er Jahre Neueingestellten (siehe Kapitel 4.4.4) hatten sich etabliert und waren als Kritiker:innen der sozialpartnerschaftlichen Linie nicht $\mathrm{zu}$ überhören. Keller, der maßgeblich an der ersten politischen Wende der GTB mitgearbeitet hatte, sah das mit Sorge, schreckte jedoch vor Enthebungen aus Funktionen, Entlassungen oder Ausschlüssen aufgrund kritischen Verhaltens dem Hauptvorstand oder dem Vorsitzenden gegenüber zurück.

Schon unter Karl Buschmann war Berthold Keller maßgeblich an der Festlegung des Kurses der GTB beteiligt gewesen. Er sicherte die politischen Entscheidungen in einem informellen Kreis ab, der sogenannten "Familie«, die bereits in den 1950er Jahren gebildet worden war und der im Kern sechs von sieben GHVMitgliedern und fünf der sieben Bezirksleiter angehörten. ${ }^{98}$ Als Vorsitzender setzte Keller keine grundlegend neuen Ideen um und selbst enge politische Freunde hätten ihn nicht unbedingt als Sympathieträger bezeichnet. Im persönlichen Umgang galt er als schwierig mit ausgeprägter cholerischer Note. Bei seinen bei-

96 CTB: Geschäftsbericht 1978-1981 des Hauptvorstandes, S. 49ff.

97 So schloss die IC Chemie 1988 kritische Vertrauensleute bei Boehringer aus ihrer Cewerkschaft aus und schloss sogar eine örtliche Verwaltungsstelle, die sich stets gegen den Kurs der Spitze gestellt hatte; vgl. Der Spiegel (1988): Cewerkschaften - Stellung unterlaufen, in: Ausgabe 32 vom 8.8.1988, S. 79-82. 1980 wurde das geschäftsführende Vorstandsmitglied Paul Plumeyer nicht zur Wiederwahl vorgeschlagen, nachdem er den Kurs des Vorstandes kritisiert hatte; vgl. Die Zeit (1980), Ausgabe 7 vom 8.2.1980.

98 Vgl. Stender (2011): Saga, S. 138. 
den Wiederwahlen erreichte er nicht das Traumergebnis seiner ersten Wahl von 91,7 Prozent, aber mit 78,7 Prozent im Jahr 1982 und 87,6 Prozent in 1986 bescherten ihm die Delegierten immer noch gute Ergebnisse.

Für das politische Innenleben der GTB war während Kellers Amtszeit nicht ohne Bedeutung, dass Gerda Linde 1986 in den Ruhestand ging. Als exponierte "Linke« hatte sie seit den 1950er Jahren die Richtungsauseinandersetzungen geführt und sich nicht gescheut, immer wieder mit ihren Kollegen im GHV in Konflikt zu gehen - und die Kollegen mit ihr. Auf Linde folgte mit Waltraud Hessedenz eine gemäßigte linksorientierte Gewerkschafterin. Geprägt von der direkten Zusammenarbeit mit den Mitgliedern an der Basis waren ihr die ideologisch bestimmten Auseinandersetzungen in der Vorstandsbürokratie fremd. Trotzdem wurde sie von Keller kritisch beäugt, insbesondere wenn sie Fortschritte in der Frauenpolitik und Frauenrepräsentanz in den Gremien einforderte oder der kritischen Jugendarbeit weitere Spielräume eröffnete.

1984 wurde Willi Arens als designierter Nachfolger für Berthold Keller in den GHV gewählt. Er stand den Ideologiestreitigkeiten eher reserviert gegenüber. Nach langjähriger Zuständigkeit von Hermann Schumacher für die Tarifpolitik wurde 1989 Wolfgang Stender aus dem Beirat in dieses Amt gewählt. Als GHVMitglied hatte Schumacher - außergewöhnlich bei den Gewerkschaften - 25 Jahre lang die Tarifpolitik verantwortet. Er agierte meist vorsichtig und in enger Abstimmung mit Bezirksleitern, ohne eigene Initiativen zu verfolgen.

Mit Wolfgang Stender bekam Schumacher einen agilen Nachfolger, der bereits die gewerkschaftsinternen Richtungskämpfe Ende der 1950er und 1960er Jahre an Berthold Kellers Seite bestritten hatte. Mit seinem ausgeprägten Selbstbewusstsein geriet er mit den Bezirksleitern und vielen Tarifkommissionsmitgliedern in Konflikt. Ähnlich wie Keller verstand er sich als politischer Kämpfer und war ebenfalls im persönlichen Umgang nicht gewinnend. Bei Stenders erster und einziger Wahl durch den Gewerkschaftstag erhielt er nur 60,3 Prozent der abgegebenen Stimmen und gab sein Amt Ende 1993 auf Druck der Bezirksleiter vorzeitig auf. ${ }^{99}$

Mit dem Hauptkassierer Hans Pfister schied Anfang 1989 ein weiterer Mitstreiter aus der Frühzeit der GTB aus dem Führungsgremium aus. Für ihn rückte Manfred Schallmeyer in den GHV auf. Als guter Rhetoriker war er zum »Liebling “ innerhalb der Organisation aufgestiegen; politisch war er anpassungsfähig. Mit seinem Wechsel in den GHV war nicht nur ein Generationenwechsel verbunden, spätestens jetzt verloren die seit den 1950er Jahren existierenden politischen Lager innerhalb der GTB an Bedeutung.

99 GTB: Protokoll des 16. Ordentlichen Cewerkschaftstages, 4.-9. November 1990 in Würzburg, S. 141. 
In der zwölfährigen Amtszeit von Berthold Keller wurden in der Textil- und der Bekleidungsindustrie 200.000 Stellen abgebaut und damit gut ein Drittel der Arbeitsplätze. Die Mitgliederverluste blieben jedoch unterproportional: Bei Kellers Ausscheiden 1990 lag der Organisationsgrad bei 47 Prozent, während er bei seinem Amtsantritt 42 Prozent betragen und zwischenzeitlich sogar die 50-Prozent-Marke überschritten hatte. Obwohl die GTB im Laufe von Kellers Amtszeit 70.000 berufstätige Mitglieder verloren hatte, lagen die jährlichen Beitragseinnahmen in Höhe von 55,4 Millionen DM um 15 Millionen DM höher als $1978 \mathrm{zu}$ Beginn seiner Amtszeit. Trotz des großen Aderlasses war die GTB bei den Beschäftigten in den Betrieben verankert, tarifpolitisch durchsetzungsfähig und finanziell stabil.

Als Berthold Keller im November 1990 altersbedingt in den Ruhestand ging, hatte sich die alte Nachkriegswelt des geteilten Deutschlands aufgelöst. Über die Folgen herrschte zu diesem Zeitpunkt große Ungewissheit, aber die GTB und ihre Führung blickte wegen der beschäftigungsstarken ostdeutschen Textil- und Bekleidungsindustrie optimistisch in die Zukunft.

Berthold Keller starb am 28. Juni 2012 im Alter von 85 Jahren in Monheim am Rhein. 